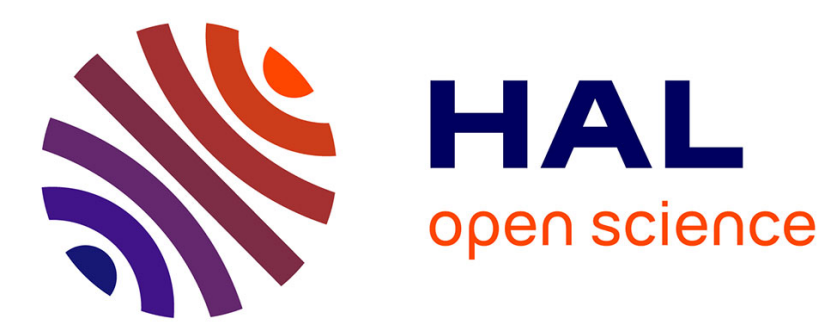

\title{
Multiscale modelling of the respiratory tract
}

Léonardo Baffico, Céline Grandmont, Bertrand Maury

\section{To cite this version:}

Léonardo Baffico, Céline Grandmont, Bertrand Maury. Multiscale modelling of the respiratory tract. Mathematical Models and Methods in Applied Sciences, 2010, pp.59-93. 10.1142/S0218202510004155 . inria-00343629v2

\section{HAL Id: inria-00343629 \\ https://hal.inria.fr/inria-00343629v2}

Submitted on 19 Mar 2009

HAL is a multi-disciplinary open access archive for the deposit and dissemination of scientific research documents, whether they are published or not. The documents may come from teaching and research institutions in France or abroad, or from public or private research centers.
L'archive ouverte pluridisciplinaire HAL, est destinée au dépôt et à la diffusion de documents scientifiques de niveau recherche, publiés ou non, émanant des établissements d'enseignement et de recherche français ou étrangers, des laboratoires publics ou privés. 


\title{
Multiscale modelling of the respiratory tract*
}

\author{
L. Baffico ${ }^{\dagger}$ \\ C. Grandmont ${ }^{\ddagger}$ \\ B. Maury ${ }^{\S}$
}

February 20, 2009

\begin{abstract}
We propose here a decomposition of the respiratory tree into three stages which correspond to different mechanical models. The resulting system is described by the Navier-Stokes equation coupled with an ODE (a simple spring model) representing the motion of the thoracic cage. We prove that this problem has at least one solution locally in time for any data and, in the special case where the spring stiffness is equal to zero, we obtain an existence result globally in time provided that the data are small enough. The behaviour of the global model is illustrated by three-dimensional simulations.
\end{abstract}

Key words : Navier-Stokes equations, local existence, coupling of models, ventilation process, Finite Element Method.

\section{Introduction, modelling aspects}

Breathing involves gas transport through the respiratory tract with its visible ends, nose and mouth. Air then streams from the pharynx down to the trachea. The trachea extends from the neck into the thorax, where it divides into right and left main bronchi, which enter the corresponding lungs. The inhaled air is then convected in the bronchus tree which ends in the alveoli embedded in a viscoelastic tissue, made in particular of blood capillaries, and where gaseous exchange occurs. Each lung is enclosed in a space bounded below by the diaphragm and laterally by the chest wall. The air movement is achieved by the displacement of the diaphragm and of the connective tissue framework of the lung (we will refer as the parenchyma in all that follows).

At the time being, the complex fractal geometry of the airway tree makes the air flow simulation on the whole tree unreachable. Besides, the distal airways from generation 7 cannot be visualized by common medical imaging techniques. Consequently, it is necessary to find new efficient strategies, including simple but realistic models. One possible choice is to try to describe the evolution of the air flux by a simple ODE as it is done in $[4,25]$. But even if the model can give valuable hints to understand the respiration mechanisms it is unsuitable to provide precise informations on the full $3 D$ flow. Our aim is to obtain a model that describes accurately the air flow in the proximal part, by

\footnotetext{
*This work has been supported by the ACINIM project Lepoumonvousdisje.

${ }^{\dagger}$ Laboratoire de mathématiques N. Oresme UMR6139, Université de Caen, BP 518614032 Caen Cedex, France

${ }^{\ddagger}$ INRIA, Projet REO Rocquencourt, BP 10578153 Le Chesnay Cedex, France

${ }^{\S}$ Laboratoire de mathématiques, Université Paris-Sud Bâtiment 425, bureau 130, 91405 Orsay Cedex, France
} 
taking into account the fact that this flow depends on the distal part and is driven by the motion of the diaphragm and the parenchyma. One solution is to find physiologically relevant boundary conditions. Yet no such "in vivo" pressure or velocity measurement is available. Thus our aim is to obtain a simplified description of the distal part. In this spirit we propose a decomposition of the respiratory tree into three stages where different models will be exploited and in which the mechanical behaviour is quite different:

- the upper part (up to the 6th generation), where the incompressible Navier-Stokes equations hold to describe the fluid flow. The flow incompressibility is valid since the Mach number in the trachea is less than 0.3 , even in forced inspiration or expiration,

- the distal part (from the 7th to the 17th generation), where one can assume that the Poiseuille law is satisfied in each bronchiole,

- the acini, where the oxygen diffusion takes place and which are embedded in an elastic medium, the parenchyma.

We will assume that the pressure is uniform in the acini part and that they are embedded in a box representing the parenchyma. The motion of the diaphragm and the parenchyma is described by a simple spring model. The decomposition can be schematized by the following figure:

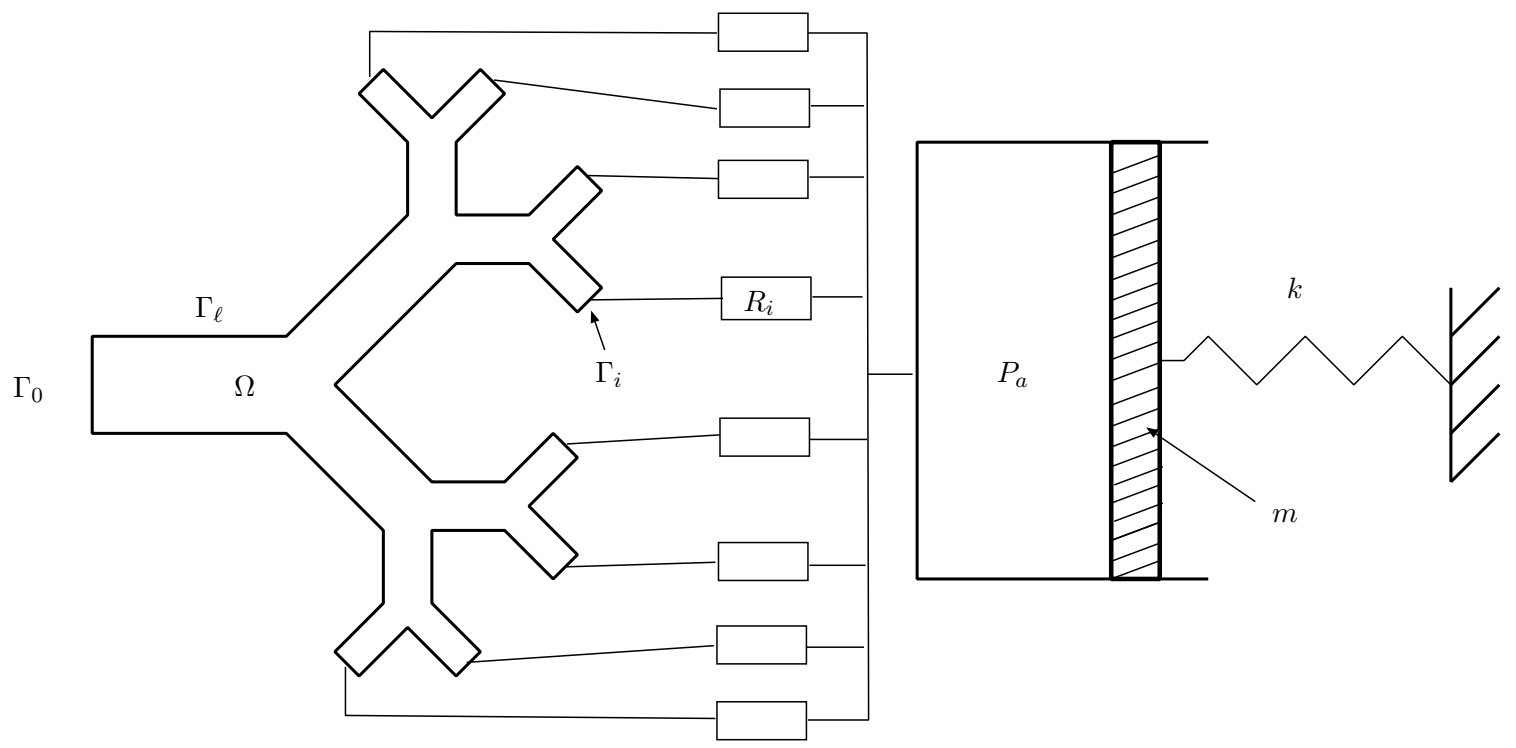

Figure 1: Multiscale model

Outlets $\Gamma_{i}, 1 \leq i \leq N$ of the upper part are coupled with Poiseuille flows, which are themselves coupled with the spring motion.

The present paper continues and generalizes the multi-model strategies proposed, for air flow modelling, in [18] or [20]. The same type of multiscale strategies has been developped and investigated for different applications such as blood flow simulations and several groups have successfully coupled three-dimensional models to either resistances or more sophisticated zero-dimensional models (lumped models) or one-dimensional models (see [15, 30, 33, 34, 38]). 
The paper is organized as follows: in the first part we present the coupled system and its variational formulation from which we derive, at least formally, an energy balance. To obtain energy estimates from this energy balance, the main difficulty is to estimate the nonlinear terms that is to say the flux, at the artificial boundaries $\Gamma_{i}$, of the system kinetic energy. For our model the energy balance leads to energy estimates only in dimension two, locally in time and for small data. Nevertheless, in the second part we prove, thanks to a Galerkin approach using a well chosen special basis, that there exists a unique smooth solution, locally in time. Moreover, in the special case where the spring stiffness is zero, we obtain that the solution exists globally in time provided the data are small enough. The proofs are based on the same kind of arguments used in [22] and rely on uniform estimates in order to pass to the limit in the discrete system. In the third part, we detail the time discretization strategy used and finally we give $3 D$ numerical evidence that this model can reproduce some aspects of normal or pathological breathing.

\section{Problem setting}

In the upper part, denoted by $\Omega$ (see Fig. 1), we assume that the Navier-Stokes equations hold:

$$
\left\{\begin{aligned}
\rho \frac{\partial \mathbf{u}}{\partial t}+\rho(\mathbf{u} \cdot \nabla) \mathbf{u}-\mu \Delta \mathbf{u}+\nabla p & =0, & & \text { in } \Omega, \\
\nabla \cdot \mathbf{u} & =0, & & \text { in } \Omega, \\
\mathbf{u} & =0, & & \text { on } \Gamma_{\ell}, \\
\mu \nabla \mathbf{u} \cdot \mathbf{n}-p \mathbf{n} & =-P_{0} \mathbf{n} & & \text { on } \Gamma_{0}, \\
\mu \nabla \mathbf{u} \cdot \mathbf{n}-p \mathbf{n} & =-\Pi_{i} \mathbf{n} & & \text { on } \Gamma_{i} \quad i=1, \ldots, N,
\end{aligned}\right.
$$

where $\mathbf{u}$ and $p$ are respectivelly the fluid velocity and the fluid pressure. On the lateral boundary $\Gamma_{\ell}$ we impose no-slip boundary conditions on the velocity, whereas on the artificial boundary $\Gamma_{i}, 0 \leq i \leq N$ we consider a pressure force exerted on the boundary. The pressure $P_{0}$ is given whereas the pressures $\Pi_{i}$ are unknown that depend on the dowstream parts. Each of the subtrees should be a dyadic tree in which we assume that the flow is laminar. Thus, by analogy with an electric network, we can consider that the flow is characterized by a unique equivalent resistance (referred to as lumped model) that depends on each resistance of the local branches (see for instance [30], [26] or [19]). Thus, each of the subtrees is replaced by a cylindrical domain, where the flow satisfies Poiseuille's law:

$$
\Pi_{i}-P_{i}=R_{i} \int_{\Gamma_{i}} \mathbf{u} \cdot \mathbf{n}, R_{i} \geq 0
$$

where $R_{i}$ denotes the equivalent resistance of the distal tree and $P_{i}$ stands for the alveolar pressure (which is supposed to be uniform). Note that $R_{i}$ depends on the geometric properties (length and diameter) of all the branches of the $i$-th subtree. Thanks to the relation (2) the boundary conditions at the outlets $\Gamma_{i}$ writes

$$
\mu \nabla \mathbf{u} \cdot \mathbf{n}-p \mathbf{n}=-P_{i} \mathbf{n}-R_{i}\left(\int_{\Gamma_{i}} \mathbf{u} \cdot \mathbf{n}\right) \mathbf{n} \text { on } \Gamma_{i} \quad i=1, \ldots, N
$$


These are non standard nonlocal boundary conditions that link the fluid stress tensor and its flux. Note that they induce dissipation in the system as we will detail it in the next section. We will call them natural dissipative boundary conditions. Note that similar conditions are also used for blood flow modelling [33, 38]. Next, we assume that all the alveola pressures are equal: $P_{i}=P_{a}$. Finally, we suppose that these alveoli are embedded in a box filled with an incompressible medium that represents the connective tissus framework of the lung. This incompressibility assumption is valid since the lung parenchyma is made mostly of elastin and collagen fibers and of blood vessels. One part of the box is connected to a spring that governs the diaphragm and parenchyma motion. The equation satisfied by the position $x$ of the diaphragm writes:

$$
m \ddot{x}=-k x+f_{\text {ext }}+f_{P},
$$

where $m$ is the total mass of the lung, $k$ is the stiffness of the spring (that characterizes the elastic behavior of the lung) and $f_{\text {ext }}$ is the force developped by the diaphragm during inspiration and forced expiration. In order to couple this simple ODE to the upper part of the model, we have to define $f_{P}$ that stands for the pressure force applied by the flow on the elastic medium. If we denote by $S$ the surface of the moving boundary box, we have

$$
f_{P}=P_{a} S .
$$

Moreover, since the flow is incompressible and since we assume that the parenchyma is made of an incompressible medium, the flow volume variation is equal to the volume variation of the parenchyma box, thus we have

$$
S \dot{x}=\sum_{i=1}^{N} \int_{\Gamma_{i}} \mathbf{u} \cdot \mathbf{n} .
$$

Note that, thanks to the fluid incompressibility, we have

$$
\sum_{i=1}^{N} \int_{\Gamma_{i}} \mathbf{u} \cdot \mathbf{n}=-\int_{\Gamma_{0}} \mathbf{u} \cdot \mathbf{n},
$$

and consequenlty

$$
S \dot{x}=-\int_{\Gamma_{0}} \mathbf{u} \cdot \mathbf{n} .
$$

Thus the coupled problem can be written as follows:

$$
\left\{\begin{array}{rlrl}
\rho \frac{\partial \mathbf{u}}{\partial t}+\rho(\mathbf{u} \cdot \nabla) \mathbf{u}-\mu \Delta \mathbf{u}+\nabla p & =0, & & \text { in }(0, T) \times \Omega, \\
\nabla \cdot \mathbf{u} & =0, & & \text { in }(0, T) \times \Omega, \\
\mathbf{u} & =0, & & \text { on }(0, T) \times \Gamma_{\ell}, \\
\mu \nabla \mathbf{u} \cdot \mathbf{n}-p \mathbf{n} & =-P_{0} \mathbf{n} & & \text { on }(0, T) \times \Gamma_{0}, \\
\mu \nabla \mathbf{u} \cdot \mathbf{n}-p \mathbf{n} & =-P_{a} \mathbf{n}-R_{i}\left(\int_{\Gamma_{i}} \mathbf{u} \cdot \mathbf{n}\right) \mathbf{n}, & & \text { on }(0, T) \times \Gamma_{i}, \\
m \ddot{x}+k x & =f_{e x t}+S P_{a}, & \\
S \dot{x} & =\sum_{i=1}^{N} \int_{\Gamma_{i}} \mathbf{u} \cdot \mathbf{n}=-\int_{\Gamma_{0}} \mathbf{u} \cdot \mathbf{n} . &
\end{array}\right.
$$


This system of equations have to be completed by suitable initial conditions

$$
\left.(\mathbf{u}, x, \dot{x})\right|_{t=0}=\left(\mathbf{u}_{0}, x_{0}, x_{1}\right), \text { with } \nabla \cdot \mathbf{u}_{0}=0, \mathbf{u}_{0}=0 \text { on } \Gamma_{\ell}, S x_{1}=-\int_{\Gamma_{0}} \mathbf{u}_{0} \cdot \mathbf{n} .
$$

One particularity of this system is that all the outlets $\Gamma_{i}, 1 \leq i \leq N$ are coupled. This is not the case, for instance, in [38] where the same type of multiscale modelling is performed but for blood flow simulations.

Remark 1.1 From a modelling standpoint, it makes sense to prescribe on $\Gamma_{0}$ also a dissipative boundary condition, to account for the resistance of the upper part of the respiratory tract (nose, pharynx, and larynx). Numerical tests (see Section 4) will be based on this assumption. As it does not change the analysis of the system, we consider here the case of a zero resistance on the inlet, to alleviate notations.

Remark 1.2 Note that the elastic behavior of the lung is described by only one degree of freedom. Moreover, in the whole coupled model we have only few parameters to fit: $m, k, S$, $f_{\text {ext }}$ and the resistances $R_{i}$. In particular by modifying $k$ and $R_{i}$ one could obtain pathological behaviors such as asthma (increase of the resistances) or emphysema (decrease of $k$ ). Nevertheless the considered spring model is a very simple one and some aspects of the respiratory cycle can not be reproduced by such a simple model, in particular the fact that the motion of the lung parenchyma is a viscolelastic media and that its motion is limited by the chest wall. We refer to [25] for a more sophisticated spring model.

Remark 1.3 By setting $\bar{p}=p-P_{a}$, by using the expression of the alveolar pressure

$$
P_{a}=\frac{m}{S} \ddot{x}+\frac{k}{S} x-\frac{f_{\text {ext }}}{S}
$$

and the volume preserving equation $S \dot{x}=-\int_{\Gamma_{0}} \mathbf{u} \cdot \mathbf{n}$, the coupled system can be written as follows:

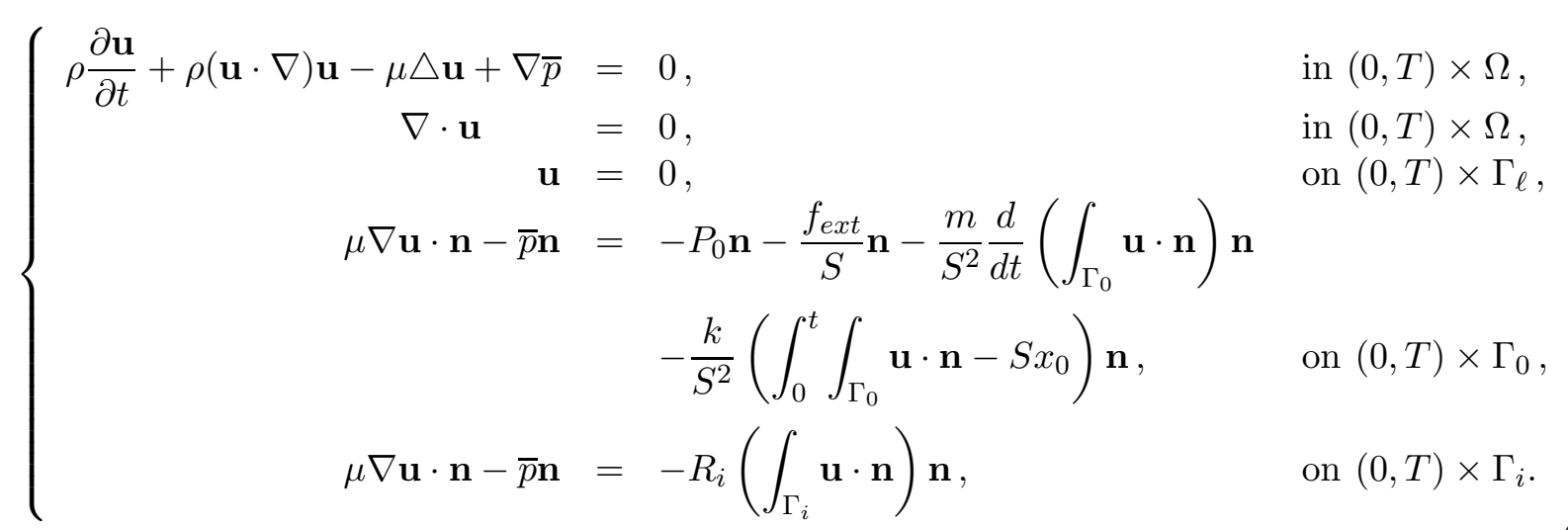

Consequently the coupled system (8) reduces to the Navier-Stokes equations, with fluid pressure replaced by the difference between fluid pressure and alveolar pressure, and with generalized natural dissipative boundary conditions. Note that the assumption of incompressiblity is essential here. 


\subsection{Variational formulation}

Assuming that all the unknowns are regular enough, we multiply the Navier-Stokes equations by a test-field $\mathbf{v}$ which vanishes on $\Gamma_{\ell}$, and the spring equation by $-(1 / S) \int_{\Gamma_{0}} \mathbf{v} \cdot \mathbf{n}$. Using

$$
x=x_{0}-\frac{1}{S} \int_{0}^{t} \int_{\Gamma_{0}} \mathbf{u} \cdot \mathbf{n},
$$

we obtain

$$
\left\{\begin{array}{l}
\rho \int_{\Omega} \partial_{t} \mathbf{u} \cdot \mathbf{v}+\rho \int_{\Omega}(\mathbf{u} \cdot \nabla) \mathbf{u} \cdot \mathbf{v}+\mu \int_{\Omega} \nabla \mathbf{u}: \nabla \mathbf{v}+\sum_{i=1}^{N} R_{i}\left(\int_{\Gamma_{i}} \mathbf{u} \cdot \mathbf{n}\right)\left(\int_{\Gamma_{i}} \mathbf{v} \cdot \mathbf{n}\right) \\
+\frac{m}{S^{2}}\left(\int_{\Gamma_{0}} \partial_{t} \mathbf{u} \cdot \mathbf{n}\right)\left(\int_{\Gamma_{0}} \mathbf{v} \cdot \mathbf{n}\right)+\frac{k}{S^{2}}\left(\int_{0}^{t} \int_{\Gamma_{0}} \mathbf{u} \cdot \mathbf{n}\right)\left(\int_{\Gamma_{0}} \mathbf{v} \cdot \mathbf{n}\right) \\
-\int_{\Omega} p \nabla \cdot \mathbf{v}+P_{a}\left(\sum_{i=1}^{N} \int_{\Gamma_{i}} \mathbf{v} \cdot \mathbf{n}-\int_{\Gamma_{0}} \mathbf{v} \cdot \mathbf{n}\right) \\
=-P_{0} \int_{\Gamma_{0}} \mathbf{v} \cdot \mathbf{n}-\frac{f_{e x t}}{S} \int_{\Gamma_{0}} \mathbf{v} \cdot \mathbf{n}+\frac{k}{S^{2}} S x_{0}\left(\int_{\Gamma_{0}} \mathbf{v} \cdot \mathbf{n}\right), \quad \forall \mathbf{v} .
\end{array}\right.
$$

Next, considering test functions $\mathbf{v}$ that are divergence free, we obtain a second variational formulation of the coupled problem

$$
\left\{\begin{array}{l}
\rho \int_{\Omega} \partial_{t} \mathbf{u} \cdot \mathbf{v}+\rho \int_{\Omega}(\mathbf{u} \cdot \nabla) \mathbf{u} \cdot \mathbf{v}+\mu \int_{\Omega} \nabla \mathbf{u}: \nabla \mathbf{v}+\sum_{i=1}^{N} R_{i}\left(\int_{\Gamma_{i}} \mathbf{u} \cdot \mathbf{n}\right)\left(\int_{\Gamma_{i}} \mathbf{v} \cdot \mathbf{n}\right) \\
+\frac{m}{S^{2}}\left(\int_{\Gamma_{0}} \partial_{t} \mathbf{u} \cdot \mathbf{n}\right)\left(\int_{\Gamma_{0}} \mathbf{v} \cdot \mathbf{n}\right)+\frac{k}{S^{2}}\left(\int_{0}^{t} \int_{\Gamma_{0}} \mathbf{u} \cdot \mathbf{n}\right)\left(\int_{\Gamma_{0}} \mathbf{v} \cdot \mathbf{n}\right) \\
=-P_{0} \int_{\Gamma_{0}} \mathbf{v} \cdot \mathbf{n}-\frac{f_{\text {ext }}}{S} \int_{\Gamma_{0}} \mathbf{v} \cdot \mathbf{n}+\frac{k}{S} x_{0}\left(\int_{\Gamma_{0}} \mathbf{v} \cdot \mathbf{n}\right), \quad \forall \mathbf{v} .
\end{array}\right.
$$

Note that here we have expressed all the quantities with the help of the fluid velocity. The velocity of the spring can be simply recovered thanks to $S \dot{x}=-\int_{\Gamma_{0}} \mathbf{u} \cdot \mathbf{n}$.

Remark 1.4 Notice that alveolar pressure can be interpreted as the Lagrange multiplier associated to the parenchyma incompressibility constraint $S \dot{x}=\sum_{i=1}^{N} \int_{\Gamma_{i}} \mathbf{u} \cdot \mathbf{n}$.

Remark 1.5 Note that in the previous variational formulation we have used the relation $\int_{\Gamma_{0}} \mathbf{u} \cdot \mathbf{n}=$ $-\sum_{i=1}^{N} \int_{\Gamma_{i}} \mathbf{u} \cdot \mathbf{n}$. This simplifies the weak formulation and will simplify the proof. However in the case where the lateral boundary $\Gamma_{l}$ is moving this equation does not hold anymore. If we do not use this 
in-flux /out-flux balance the variational formulation writes:

$$
\left\{\begin{array}{l}
\rho \int_{\Omega} \partial_{t} \mathbf{u} \cdot \mathbf{v}+\rho \int_{\Omega}(\mathbf{u} \cdot \nabla) \mathbf{u} \cdot \mathbf{v}+\mu \int_{\Omega} \nabla \mathbf{u}: \nabla \mathbf{v}+\sum_{i=1}^{N} R_{i}\left(\int_{\Gamma_{i}} \mathbf{u} \cdot \mathbf{n}\right)\left(\int_{\Gamma_{i}} \mathbf{v} \cdot \mathbf{n}\right) \\
+\frac{m}{S^{2}}\left(\sum_{i=1}^{N} \int_{\Gamma_{i}} \partial_{t} \mathbf{u} \cdot \mathbf{n}\right)\left(\sum_{i=1}^{N} \int_{\Gamma_{i}} \mathbf{v} \cdot \mathbf{n}\right)+\frac{k}{S^{2}}\left(\int_{0}^{t} \sum_{i=1}^{N} \int_{\Gamma_{i}} \mathbf{u} \cdot \mathbf{n}\right)\left(\sum_{i=1}^{N} \int_{\Gamma_{i}} \mathbf{v} \cdot \mathbf{n}\right) \\
=-P_{0} \int_{\Gamma_{0}} \mathbf{v} \cdot \mathbf{n}-\frac{f_{\text {ext }}}{S} \sum_{i=1}^{N} \int_{\Gamma_{i}} \mathbf{v} \cdot \mathbf{n}+\frac{k}{S} x_{0}\left(\sum_{i=1}^{N} \int_{\Gamma_{i}} \mathbf{v} \cdot \mathbf{n}\right), \quad \forall \mathbf{v} .
\end{array}\right.
$$

Note that the existence of solution as well as the numerical simulations can be performed also in this case with only a few changes which we will underline in what follows (see remarks 2.5 and 3.2).

\subsection{Energy balance}

We start to derive, at least formally, an energy balance for the coupled system. We take $\mathbf{v}=\mathbf{u}$ as a test function in (12). By writing the convective terms, thanks to an integration by parts, as a flux of kinetic energy at the inlet and at the outlets, and remembering that $S \dot{x}=-\int_{\Gamma_{0}} \mathbf{u} \cdot \mathbf{n}$, we obtain

$$
\begin{gathered}
\quad \frac{d}{d t} \underbrace{\left(\frac{\rho}{2} \int_{\Omega}|\mathbf{u}|^{2}+\frac{m}{2}|\dot{x}|^{2}+\frac{k}{2}|x|^{2}\right)}_{\text {Total energy }}+\underbrace{\mu \int_{\Omega}|\nabla \mathbf{u}|^{2}}_{\text {Dissipation within } \Omega}+\underbrace{\sum_{i=1}^{N} R_{i}\left(\int_{\Gamma_{i}} \mathbf{u} \cdot \mathbf{n}\right)^{2}}_{\text {Dissipation in the subtrees }} \\
=-\underbrace{\frac{\rho}{2} \sum_{i=0}^{N} \int_{\Gamma_{i}}|\mathbf{u}|^{2}(\mathbf{u} \cdot \mathbf{n})}_{\text {In/out-come of kinetic energy }}+\underbrace{P_{0} S \dot{x}}_{\text {Power of inlet pressure }}+\underbrace{f_{\text {ext }} \dot{x}}_{\text {Power of ext. forces }} .
\end{gathered}
$$

Identity (14) represents the energy balance of the coupled system. The two first terms represent the variation of the total kinetic energy, the third one being the variation of the mechanical energy of the spring. Moreover, the energy is dissipated: as for the standard Navier-Stokes equations the fact that the flow is viscous contribute to the dissipation of the energy but here there is a second contribution to the dissipation that comes from the resistive part of the bronchial tree, namely

$$
\sum_{i=1}^{N} R_{i}\left(\int_{\Gamma_{i}} \mathbf{u} \cdot \mathbf{n}\right)^{2} .
$$

This energy balance will provide energy estimates in the case where the flux of kinetic energy at the interface can be correctly estimated by the global kinetic energy and the dissipated energy. Unfortunatly we can control the flux of kinetic energy only locally in time and for small data and only in the case where $d=2$. Note that in [18] this difficulty has been overcome by considering other type of boundary conditions at the inlet and outlets. More precisely the boundary conditions do not involve the fluid stress tensor but the total fluid stress tensor, i.e. where the fluid pressure $p$ has been 
replaced by the total pressure $p+\rho \frac{|\mathbf{u}|^{2}}{2}$. Consequently, the flux of kinetic energy on the boundary do not appear in the energy balance. An alternative approach is proposed in [20], where a more suited estimate of the nonlinear convective term is obtained thanks to an additionnal assumption that states that the velocity profiles at the inlet and outlets are given. Note that this estimate relies on the fact that the trace of the fluid velocity on each $\Gamma_{i}$ is supposed to be described by a single parameter.

\section{Well-posedness issues}

\subsection{Mathematical setting, notations}

In this section we put the variational formulation (12) onto an abstract form, on which we shall base the well-posedness proof.

Let $\Omega$ be a bounded domain in $\mathbb{R}^{d}, d=2$ or $3, \Gamma_{0}$ the inlet boundary, $\Gamma_{i}$ the $i$-th. outlet, and $\Gamma_{\ell}$ the lateral boundary (see Fig. 1). We introduce the following functional spaces:

$$
\begin{gathered}
V=\left\{\mathbf{v} \in H^{1}(\Omega)^{d}, \nabla \cdot \mathbf{v}=0, \mathbf{v}=0 \text { on } \Gamma_{\ell}\right\} . \\
H=\bar{V}^{L^{2}},
\end{gathered}
$$

We denote by $(\cdot, \cdot)_{0}$ the scalar product on $H \times H$ defined by

$$
(\mathbf{v}, \mathbf{w})_{0}=\rho \int_{\Omega} \mathbf{v} \cdot \mathbf{w}+\frac{m}{S^{2}}\left(\int_{\Gamma_{0}} \mathbf{v} \cdot \mathbf{n}\right)\left(\int_{\Gamma_{0}} \mathbf{w} \cdot \mathbf{n}\right),
$$

and by $\|\cdot\|_{0}$ the associated norm (see Remark 2.4 below for a proper definition of the flux $\int_{\Gamma_{0}} \mathbf{v} \cdot \mathbf{n}$ for $\mathbf{v} \in H)$. Next we set

$$
a_{1}(\mathbf{v}, \mathbf{w})=\mu \int_{\Omega} \nabla \mathbf{v}: \nabla \mathbf{w}+\sum_{i=1}^{N} R_{i}\left(\int_{\Gamma_{i}} \mathbf{v} \cdot \mathbf{n}\right)\left(\int_{\Gamma_{i}} \mathbf{w} \cdot \mathbf{n}\right)
$$

and

$$
b(\mathbf{u}, \mathbf{v}, \mathbf{w})=\rho \int_{\Omega}(\mathbf{u} \cdot \nabla) \mathbf{v} \cdot \mathbf{w},
$$

that are the bilinear and trilinear forms on $V$ associated to our system. Moreover we introduce

$$
a_{2}(\mathbf{v}, \mathbf{w})=\frac{k}{S^{2}}\left(\int_{\Gamma_{0}} \mathbf{v} \cdot \mathbf{n}\right)\left(\int_{\Gamma_{0}} \mathbf{w} \cdot \mathbf{n}\right) .
$$

Finally we introduce an operator $A$ whose eigenvectors will constitute the Galerkin basis used to build our sequence of approximate solutions.

Definition 2.1 (Operator A).

The operator $A$ is defined on $H$ as follows:

$$
\begin{gathered}
D(A)=\left\{\mathbf{v} \in V,\left|a_{1}(\mathbf{v}, \mathbf{w})\right| \leq C\|\mathbf{v}\|_{0}, \forall \mathbf{w} \in V\right\}, \\
(A \mathbf{v}, \mathbf{w})_{0}=a_{1}(\mathbf{v}, \mathbf{w}), \forall(\mathbf{v}, \mathbf{w}) \in D(A) \times V .
\end{gathered}
$$


Remark 2.2 Operator $A$ is similar to Stokes operator, with special kinds of boundary conditions. More precisely, one can check that $A \mathbf{u}=\mathbf{f}$ if and only if there exists a pressure field $p$ over $\Omega$ such that $(\mathbf{u}, p)$ is a weak solution to the Stokes problem

$$
\left\{\begin{aligned}
-\mu \Delta \mathbf{u}+\nabla p & =\mathbf{f} & & \text { in } \Omega \\
\nabla \cdot \mathbf{u} & =0 & & \text { in } \Omega \\
\mathbf{u} & =0 & & \text { on } \Gamma_{\ell}, \\
\mu \nabla \mathbf{u} \cdot \mathbf{n}-p \mathbf{n} & =\left(\frac{m}{S^{2}} \int_{\Gamma_{0}} \mathbf{f} \cdot \mathbf{n}\right) \mathbf{n} & & \text { on } \Gamma_{0}, \\
\mu \nabla \mathbf{u} \cdot \mathbf{n}-p \mathbf{n} & =-R_{i}\left(\int_{\Gamma_{i}} \mathbf{u} \cdot \mathbf{n}\right) \mathbf{n} & & \text { on } \Gamma_{i}, i=1, \ldots, N .
\end{aligned}\right.
$$

We may now write the variational formulation in an abstract setting, on which we base the notion of solution to our problem.

Definition 2.3 We shall say that $\mathbf{u}$ is a solution of (8)-(9) on $[0, T]$ if

$$
\left\{\begin{array}{l}
\mathbf{u} \in L^{2}(0, T ; D(A)) \cap L^{\infty}(0, T ; V) \cap H^{1}(0, T ; H), \\
\frac{d}{d t}(\mathbf{u}, \mathbf{v})_{0}+a_{1}(\mathbf{u}, \mathbf{v})+b(\mathbf{u}, \mathbf{u}, \mathbf{v})+a_{2}\left(\int_{0}^{t} \mathbf{u}, \mathbf{v}\right)=\ell(\mathbf{v}) \quad \forall \mathbf{v} \in V,
\end{array}\right.
$$

with $\ell(\mathbf{v})=-P_{0} \int_{\Gamma_{0}} \mathbf{v} \cdot \mathbf{n}-\frac{f_{\text {ext }}}{S} \int_{\Gamma_{0}} \mathbf{v} \cdot \mathbf{n}+\frac{k}{S^{2}} S x_{0}\left(\int_{\Gamma_{0}} \mathbf{v} \cdot \mathbf{n}\right)$, and initial condition $\mathbf{u}(0)=\mathbf{u}_{0}$.

Remark 2.4 The flux term $\int_{\Gamma_{0}} \mathbf{v} \cdot \mathbf{n}$ in (15) can be defined for $\mathbf{v}$ in $H$ by mean of the standard duality as

$$
\langle\mathbf{v} \cdot \mathbf{n}, g\rangle_{H^{-1 / 2}(\partial \Omega), H^{1 / 2}(\partial \Omega)}=\int_{\Omega} \mathbf{v} \cdot \nabla g,
$$

where $g$ is any function in $H^{1}(\Omega)$ such that $g=1$ on $\Gamma_{0}$, and which vanishes on $\Gamma_{i}$ for $i=1, \ldots, N$. Such a function exists since in- and out-let boundaries $\Gamma_{i}$ are not in contact. Note that if $\mathbf{v} \in V$ then the provided definition corresponds to the flux of $\mathbf{v}$ through $\Gamma_{0}$, because, on the lateral boundary $\Gamma_{\ell}$, $\mathbf{v} \cdot \mathbf{n}$ vanishes, thus

$$
\langle\mathbf{v} \cdot \mathbf{n}, g\rangle_{H^{-1 / 2}(\partial \Omega), H^{1 / 2}(\partial \Omega)}=\int_{\Gamma_{0}} \mathbf{v} \cdot \mathbf{n} .
$$

We can define in the same way the velocity flux $\int_{\Gamma_{i}} \mathbf{v} \cdot \mathbf{n}$ on $\Gamma_{i}$ for $\mathbf{v} \in H$.

Remark 2.5 If we had chosen to work with the variational formulation (13) then the scalar product on $H$ and the bilinear form associated to the spring energy have to be modified respectivelly as follows:

$$
(\mathbf{v}, \mathbf{w})_{0}=\rho \int_{\Omega} \mathbf{v} \cdot \mathbf{w}+\frac{m}{S^{2}}\left(\sum_{i-1}^{N} \int_{\Gamma_{i}} \mathbf{v} \cdot \mathbf{n}\right)\left(\sum_{i-1}^{N} \int_{\Gamma_{i}} \mathbf{w} \cdot \mathbf{n}\right),
$$




$$
a_{2}(\mathbf{v}, \mathbf{w})=\frac{k}{S^{2}}\left(\sum_{i-1}^{N} \int_{\Gamma_{i}} \mathbf{v} \cdot \mathbf{n}\right)\left(\sum_{i-1}^{N} \int_{\Gamma_{i}} \mathbf{w} \cdot \mathbf{n}\right) .
$$

The study of the operator $A$ is then very similar and the proof of existence can be easily adapted to this case.

\subsection{Preliminaries}

We gather here some properties, mainly pertaining to the operator $A$ which we introduced in the previous section. We begin with an estimate for divergence free fields that will be essential to control boundary integrals:

\section{Lemma 2.6 It holds}

$$
\left|\int_{\Gamma_{0}} \mathbf{v} \cdot \mathbf{n}\right| \leq C\|\mathbf{v}\|_{L^{2}(\Omega)}, \quad \forall \mathbf{v} \in H
$$

where the boundary integral is defined according to Remark 2.4. As a consequence, $\|\cdot\|_{0}$ is equivalent to the $L^{2}$ norm on $H$.

This a direct consequence of the definition of $\left(H^{-1 / 2}, H^{1 / 2}\right)$-duality, based on the Green formula:

$$
\int_{\Gamma_{0}} \mathbf{v} \cdot \mathbf{n}:=\langle\mathbf{v} \cdot \mathbf{n}, g\rangle_{H^{-1 / 2}(\partial \Omega), H^{1 / 2}(\partial \Omega)}=\int_{\Omega} g \nabla \cdot \mathbf{v}+\int_{\Omega} \mathbf{v} \cdot \nabla g
$$

where $g \in H^{1}(\Omega)$ is 1 on $\Gamma_{0}$, and vanishes on $\Gamma_{i}$ for $i=1, \ldots, N$.

Proposition 2.7 The operator A has the following properties:

i) $A \in \mathcal{L}(D(A), H)$ is invertible and its inverse is compact on $H$.

ii) $A$ is selfadjoint.

As a consequence, $A$ admits a family of eigenfunctions $\left(\phi_{j}\right)$

$$
A \phi_{j}=\lambda_{j} \phi_{j} \text { with } 0<\lambda_{1} \leq \lambda_{2} \leq \ldots \quad \lambda_{j} \underset{j \rightarrow+\infty}{\longrightarrow}+\infty,
$$

which is complete and orthogonal in both $H$ and $V$.

Proof: The proof of this proposition relies on classical arguments (see for instance [6]).

As in [22] (or in [33]), the control of the nonlinear term in a priori estimates is based on regularity properties of operator $A$ :

Proposition 2.8 Let $\Omega$ be a domain in $\mathbb{R}^{d}$. We suppose that $\Omega$ is piecewise smooth (in-/out-let and lateral component of the boundary are smooth, see Fig. 1), and that the $\Gamma_{i}$ 's meet with lateral boundary $\Gamma_{\ell}$ at angle $\pi / 2$. Let $A$ be defined by Def. 2.1. There exists $\varepsilon>0$ and a constant $C$ such that, for any $\mathbf{f} \in H, A \mathbf{u}=\mathbf{f}$, it holds

$$
\|\mathbf{u}\|_{H^{3 / 2+\varepsilon}(\Omega)} \leq C\|\mathbf{f}\|_{0}
$$


Proof: First of all, as $\mathbf{f}$ is in $H$, it is divergence free, so that

$$
\left|\int_{\Gamma_{i}} \mathbf{f} \cdot \mathbf{n}\right| \leq C\|\mathbf{f}\|_{0}
$$

By Remark 2.2, u solves Stokes problem with right-hand side f, homogeneous Dirichlet boundary conditions on the lateral boundaries, dissipative conditions on the $\Gamma_{i}^{\prime} s$, and free boundary conditions on the inlet $\Gamma_{0}$ with an oustide pressure which is controlled by the normal flux of $\mathbf{f}$. By taking $\mathbf{u}$ as a test function, and using (19), we have

$$
\|\mathbf{u}\|_{H^{1}(\Omega)} \leq C\|\mathbf{f}\|_{0} .
$$

Now consider a auxilliary field $\tilde{p} \in H^{1}(\Omega)$ defined as follows: It is harmonic over $\Omega$,

$$
\tilde{p}=\left(\frac{m}{S^{2}} \int_{\Gamma_{0}} \mathbf{f} \cdot \mathbf{n}\right) \text { on } \Gamma_{0}, \tilde{p}=-R_{i}\left(\int_{\Gamma_{i}} \mathbf{u} \cdot \mathbf{n}\right) \text { on } \Gamma_{i}, i=1, \ldots, N, \text { and } \frac{\partial \tilde{p}}{\partial n}=0 \text { on } \Gamma_{\ell} .
$$

As normal fluxes of $\mathbf{u}$ and $\mathbf{f}$ are both controlled by $\|\mathbf{f}\|_{0}$, so is the $H^{1}$ norm of $\tilde{p}$. Problem (17) can therefore by written

$$
\left\{\begin{aligned}
-\mu \triangle \mathbf{u}+\nabla(p-\tilde{p}) & =\rho \mathbf{f}-\nabla \tilde{p} & & \text { in } \Omega \\
\nabla \cdot \mathbf{u} & =0 & & \text { in } \Omega \\
\mathbf{u} & =0 & & \text { on } \Gamma_{\ell}, \\
\mu \nabla \mathbf{u} \cdot \mathbf{n}-(p-\tilde{p}) \mathbf{n} & =0 & & \text { on } \Gamma_{0}, \\
\mu \nabla \mathbf{u} \cdot \mathbf{n}-(p-\tilde{p}) \mathbf{n} & =0 & & \text { on } \Gamma_{i}, i=1, \ldots, N .
\end{aligned}\right.
$$

which is a standard Stokes problem with free out/in-let boundary conditions at the ends, and a new right hand-side with a $L^{2}$ norm controlled by $\|\mathbf{f}\|_{0}$. As $H^{2}$ regularity is not an issue away from the $\Gamma_{i}^{\prime} s$, we end up with a question of regularity for Stokes problem in a single pipe, with free outlet B.C.'s and $L^{2}$ right-hand side.

This regularity is a consequence of recent regularity results for the Stokes problem in nonsmooth domains, for mixed boundary conditions. In the two-dimensional setting, the $H^{3 / 2+\varepsilon}$ regularity of $\mathbf{u}$, and continuous dependance upon the data $f$, is given in [32]. For the three dimensionnal problem, the key point lies in some weighted estimates for the Stokes problem in a dihedron, which we describe below. We shall disregard here any considerations for conditions at infinity, as they do not play any role in our case.

Up to a $C^{\infty}$ diffeomorphism, the domain is locally similar quarter-space like domain. Therefore we consider a dihedral domain $\Omega$ delimited by flat hypersurfaces $\Gamma_{1}$ and $\Gamma_{2}$, which meet at angle $\theta$, and $(\mathbf{w}, q)$ the solution to

$$
\begin{cases}-\Delta \mathbf{w}+\nabla q & =\mathbf{g} \\ \nabla \cdot \mathbf{w} & =0\end{cases}
$$

with homogeneous boundary conditions

$$
\nabla \mathbf{w} \cdot \mathbf{n}-q \mathbf{n}=0 \quad \text { on } \Gamma_{1}, \mathbf{w}=0 \quad \text { on } \Gamma_{2} .
$$


Lemma 2.16 in [28] asserts that $\mathbf{u}$ is in some weighted Sobolev space $W_{\delta}^{2}(\Omega)$, with

$$
\|\mathbf{w}\|_{W_{\delta}^{2}} \leq C\|\mathbf{g}\|_{0}
$$

as soon as none of the real parts of the solutions to

$$
\cos (\lambda \theta)\left(\lambda^{2} \sin ^{2} \theta-\cos ^{2}(\lambda \theta)\right)=0
$$

fits into $[0,1-\delta]$. Considering $\theta=\pi / 2$, the solution to the previous equation with the smallest positive real part is $\lambda_{c} \approx 0.59$. As a consequence, $\delta=1 / 2-\varepsilon$ qualifies as soon as $\varepsilon$ is sufficiently small $(\varepsilon<0.09)$.

The norm on $W_{\delta}^{2}$ is defined by

$$
\|\mathbf{w}\|_{W_{\delta}^{2}}^{2}=\sum_{|\alpha| \leq 2} \sum_{1 \leq i \leq 3} \int_{\Omega} r^{2 \delta}\left|\partial_{\alpha} w_{i}\right|^{2},
$$

where $r$ is the distance to the edge of $\Omega$. The expected estimate is a consequence of a Hardy type inequality (see Proposition A.1 in the appendix):

$$
|u|_{\dot{H}^{2-\delta}} \leq C\|u\|_{W_{\delta}^{2}}^{2},
$$

which yields the expected estimate for $\delta=1 / 2-\varepsilon$, in the case of a quarter-space domain.

Corollary 2.9 Under the assumptions of Proposition 2.8, the following inequality holds

$$
\|\mathbf{v}\|_{L^{\infty}(\Omega)} \leq C\|A \mathbf{v}\|_{0},
$$

and there exists $\theta \in(0,1)$ such that

$$
\|\mathbf{v}\|_{L^{\infty}(\Omega)} \leq C\|\nabla \mathbf{v}\|_{L^{2}(\Omega)}^{\theta}\|A \mathbf{v}\|_{0}^{1-\theta} .
$$

Proof: The first estimate is a direct consequence of $H^{3 / 2+\varepsilon}(\Omega) \hookrightarrow L^{\infty}(\Omega)$. As for the second estimate, we use the interpolation estimate (for $0<\varepsilon^{\prime}<\varepsilon$ ),

$$
\|\mathbf{v}\|_{H^{3 / 2+\varepsilon^{\prime}}(\Omega)} \leq C\|\nabla \mathbf{v}\|_{L^{2}(\Omega)}^{\theta}\|A \mathbf{v}\|_{H^{3 / 2+\varepsilon}(\Omega)}^{1-\theta},
$$

valid for $\theta$ such that $3 / 2+\varepsilon^{\prime}=\theta+(1-\theta)(3 / 2+\varepsilon)$.

\subsection{Existence and uniqueness of a solution}

We establish here two types of existence results: one locally in time for large data, and one globally in time for small enough data in the special case where the stiffness of the spring is equal to zero.

Let us first make explicit our assumptions:

$\Omega$ is piecewise regular in the sense of Proposition 2.8, which is assumed to hold true

$$
T>0, \quad P_{0} \in L^{2}(0, T), \quad f_{\text {ext }} \in L^{2}(0, T), \mathbf{u}_{0} \in V, R_{i} \geq 0, i=1, \ldots, N .
$$


Theorem 2.10 Under assumptions (21)-(22), there exists a time interval $\left[0, t^{\star}\right]$ on which (8)-(9) has a unique solution in the sense of Def. 2.3. Moreover, in the special case where $k=0$ and for small data (initial conditions and applied forces small enough in norm $L^{\infty}$ in time), then the solution can be defined globally in time, and there exists $C>0$ such that for all $t>0$

$$
\|\nabla \mathbf{u}\|_{L^{2}(\Omega)}^{2}+\sum_{i=1}^{N} R_{i}\left(\int_{\Gamma_{i}} \mathbf{u} \cdot \mathbf{n}\right)^{2} \leq C .
$$

Proof: We follow the same steps as in [22]. First we build a sequence of approximated solutions thanks to a Galerkin method. Then, we derive uniform bounds that enable us to pass to the limit in the equation leading to the existence of at least one solution of (18). Finally we prove that this solution is unique.

Remark 2.11 In [22] or [33], the existence of a unique smooth solution of the Navier-Stokes system is proved for other types of boundary conditions, namely mean pressure or mean flux boundary conditions ([22]) or boundary conditions that write $\mu \nabla \mathbf{u} \cdot \mathbf{n}-p \mathbf{n}+R_{i}(\mathbf{u} \cdot \mathbf{n}) \mathbf{n}=\mathbf{g}$ ([33]). Note that in the former, the existence is obtained under a condition on the size of the $R_{i}$ 's coefficients which we do not need here, thanks to the choice of the operator $A$ (that takes into account the dissipative part coming from the subtrees resistances). Moreover in [33] the existence of a solution of the Navier-Stokes system coupled with lumped models is proved thanks to a fixed point argument. Note that we can not use here the same kind of argument due to the nature of the coupling. That is the reason why we have considered a non standard scalar product on $H$ taking into account the spring mass in order to treat the system globally.

Galerkin approximation. For all $n \in \mathbb{N}$, we define the following discrete problem: Find $\mathbf{u}_{n}(t) \in \operatorname{span}\left(\phi_{1}, \ldots, \phi_{n}\right)$ such that

$$
\left\{\begin{array}{l}
\left(\frac{\partial \mathbf{u}_{n}}{\partial t}, \mathbf{v}\right)_{0}+a_{1}\left(\mathbf{u}_{n}, \mathbf{v}\right)+b\left(\mathbf{u}_{n}, \mathbf{u}_{n}, \mathbf{v}\right)+a_{2}\left(\int_{0}^{t} \mathbf{u}_{n}, \mathbf{v}\right)=\ell(\mathbf{v}), \quad \forall \mathbf{v} \in \operatorname{span}\left(\phi_{1}, \ldots, \boldsymbol{\phi}_{n}\right), \\
\left(\mathbf{u}_{n}(0)-\mathbf{u}_{0}, \mathbf{v}\right)_{0}=0, \quad \forall \mathbf{v} \in \operatorname{span}\left(\phi_{1}, \ldots, \boldsymbol{\phi}_{n}\right) .
\end{array}\right.
$$

The first step consists in proving that this differential system has a unique solution on a time interval $\left[0, t^{\star}\right]$, where $t^{\star}$ does not depend on $n$. Let us denote by $t_{n}$ the life-time of the maximal solution $\mathbf{u}_{n}$ over $[0, T]$.

A priori estimates. In what follows, $C$ stands for a generic constant whose value may change, but it does not depend on $n$. We write (23) for the test function $\mathbf{v}=A \mathbf{u}_{n}$, which is admissible thanks to the choice of the Galerkin basis. Indeed $A \mathbf{u}_{n} \in \operatorname{span}\left(\phi_{1}, \ldots, \phi_{n}\right)$. Taking into account the two following equalities

$$
\left(\frac{\partial \mathbf{u}_{n}}{\partial t}, A \mathbf{u}_{n}\right)_{0}=\mu \frac{1}{2} \frac{d}{d t}\left\|\nabla \mathbf{u}_{n}\right\|_{L^{2}(\Omega)}^{2}+\frac{1}{2} \sum_{i=1}^{N} R_{i} \frac{d}{d t}\left(\int_{\Gamma_{i}} \mathbf{u}_{n} \cdot \mathbf{n}\right)^{2}
$$


and

$$
a_{1}\left(\mathbf{u}_{n}, A \mathbf{u}_{n}\right)=\left\|A \mathbf{u}_{n}\right\|_{0}^{2},
$$

we obtain

$$
\begin{aligned}
& \frac{1}{2} \mu \frac{d}{d t}\left\|\nabla \mathbf{u}_{n}\right\|_{L^{2}(\Omega)}^{2}+\frac{1}{2} \sum_{i=1}^{N} R_{i} \frac{d}{d t}\left(\int_{\Gamma_{i}} \mathbf{u}_{n} \cdot \mathbf{n}\right)^{2}+\left\|A \mathbf{u}_{n}\right\|_{0}^{2} \\
= & -b\left(\mathbf{u}_{n}, \mathbf{u}_{n}, A \mathbf{u}_{n}\right)-a_{2}\left(\int_{0}^{t} \mathbf{u}_{n}, A \mathbf{u}_{n}\right)+\ell\left(A \mathbf{u}_{n}\right) .
\end{aligned}
$$

Now thanks to Corollary 2.9 and Lemma 2.6, we can control the nonlinear term:

$$
\begin{aligned}
\left|b\left(\mathbf{u}_{n}, \mathbf{u}_{n}, A \mathbf{u}_{n}\right)\right| & \leq C\left\|\mathbf{u}_{n}\right\|_{L^{\infty}(\Omega)}\left\|\nabla \mathbf{u}_{n}\right\|_{L^{2}(\Omega)}\left\|A \mathbf{u}_{n}\right\|_{L^{2}(\Omega)} \\
& \leq C\left\|\nabla \mathbf{u}_{n}\right\|_{L^{2}(\Omega)}^{1+\theta}\left\|A \mathbf{u}_{n}\right\|_{0}^{2-\theta} \quad \text { with } \theta \in(0,1) \\
& \leq C\left\|\nabla \mathbf{u}_{n}\right\|_{L^{2}(\Omega)}^{2(1+\theta) / \theta}+\frac{1}{4}\left\|A \mathbf{u}_{n}\right\|_{0}^{2},
\end{aligned}
$$

by Young's inequality. Moreover, using Lemma 2.6, the equivalence of the $L^{2}$ norm and $\|\cdot\|_{0}$ on $H$ and Poincaré inequality, we obtain

$$
\begin{aligned}
\left|a_{2}\left(\int_{0}^{t} \mathbf{u}_{n}, A \mathbf{u}_{n}\right)\right| & =k\left|\left(\int_{0}^{t} \int_{\Gamma_{0}} \mathbf{u}_{n} \cdot \mathbf{n}\right)\left(\int_{\Gamma_{0}} A \mathbf{u}_{n} \cdot \mathbf{n}\right)\right| \\
& \leq C k \int_{0}^{t}\left\|\nabla \mathbf{u}_{n}\right\|_{L^{2}(\Omega)}^{2}+\frac{1}{4}\left\|A \mathbf{u}_{n}\right\|_{0}^{2},
\end{aligned}
$$

and

$$
\left|\ell\left(A \mathbf{u}_{n}\right)\right| \leq C\left(\left|P_{0}\right|^{2}+\frac{\left|f_{e x t}\right|^{2}}{S^{2}}+\frac{k^{2}\left|x_{0}\right|^{2}}{S^{2}}\right)+\frac{1}{4}\left\|A \mathbf{u}_{n}\right\|_{0}^{2}
$$

Consequently

$$
\begin{aligned}
& \frac{1}{2} \mu \frac{d}{d t}\left\|\nabla \mathbf{u}_{n}\right\|_{L^{2}(\Omega)}^{2}+\frac{1}{2} \sum_{i=1}^{N} R_{i} \frac{d}{d t}\left(\int_{\Gamma_{i}} \mathbf{u}_{n} \cdot \mathbf{n}\right)^{2}+\frac{1}{4}\left\|A \mathbf{u}_{n}\right\|_{0}^{2} \\
& \leq C\left\|\nabla \mathbf{u}_{n}\right\|_{L^{2}(\Omega)}^{2(1+\theta) / \theta}+C\left(\left|P_{0}\right|^{2}+\frac{\left|f_{\text {ext }}\right|^{2}}{S^{2}}+\frac{k^{2}\left|x_{0}\right|^{2}}{S^{2}}\right)+k C \int_{0}^{t}\left\|\nabla \mathbf{u}_{n}\right\|_{L^{2}(\Omega)}^{2} .
\end{aligned}
$$

Next we set

$$
\begin{gathered}
\Phi_{n}(t)=\mu\left\|\nabla \mathbf{u}_{n}\right\|_{L^{2}(\Omega)}^{2}+\sum_{i=1}^{N} R_{i}\left(\int_{\Gamma_{i}} \mathbf{u}_{n} \cdot \mathbf{n}\right)^{2}+k \int_{0}^{t}\left\|\nabla \mathbf{u}_{n}\right\|_{L^{2}(\Omega)}^{2} \\
\Psi_{n}(t)=\frac{1}{2}\left\|A \mathbf{u}_{n}\right\|_{0}^{2}
\end{gathered}
$$

and

$$
f(t)=C\left(\left|P_{0}\right|^{2}+\frac{\left|f_{e x t}\right|^{2}}{S^{2}}+\frac{k^{2}\left|x_{0}\right|^{2}}{S^{2}}\right) .
$$


Then, by adding $\frac{k}{2}\left\|\nabla \mathbf{u}_{n}\right\|_{L^{2}(\Omega)}^{2}$ on both sides, (24) writes

$$
\frac{d}{d t} \Phi_{n}(t)+\Psi_{n}(t) \leq C\left(\Phi_{n}^{(1+\theta) / \theta}(t)+\Phi_{n}(t)\right)+f(t)
$$

Besides, the choice of the initial condition

$$
\left(\mathbf{u}_{n}(0)-\mathbf{u}_{0}, \mathbf{v}\right)_{0}=0, \quad \forall \mathbf{v} \in \operatorname{span}\left(\phi_{1}, \cdots, \phi_{n}\right),
$$

implies, choosing $\mathbf{v}=A \mathbf{u}_{n}(0)$,

$$
\left(\mathbf{u}_{n}(0), A \mathbf{u}_{n}(0)\right)_{0}=\left(\mathbf{u}_{0}, A \mathbf{u}_{n}(0)\right)_{0}
$$

which yields

$$
a_{1}\left(\mathbf{u}_{n}(0), \mathbf{u}_{n}(0)\right)=a_{1}\left(\mathbf{u}_{n}(0), \mathbf{u}_{0}\right) .
$$

Thus, Cauchy-Schwarz inequality implies

$$
\Phi_{n}(0)=\mu\left\|\nabla \mathbf{u}_{n}(0)\right\|_{L^{2}(\Omega)}^{2}+\sum_{i=1}^{N} R_{i}\left(\int_{\Gamma_{i}} \mathbf{u}_{n}(0) \cdot \mathbf{n}\right)^{2} \leq \mu\left\|\nabla \mathbf{u}_{0}\right\|_{L^{2}(\Omega)}^{2}+\sum_{i=1}^{N} R_{i}\left(\int_{\Gamma_{i}} \mathbf{u}_{0} \cdot \mathbf{n}\right)^{2} .
$$

Let us denote by $t^{\star} \leq T$ a positive time such that the solution $F$ to

$$
F^{\prime}=C\left(F^{(1+\theta) / \theta}+F\right)+f, F(0)=\mu\left\|\nabla \mathbf{u}_{0}\right\|_{L^{2}(\Omega)}^{2}+\sum_{i=1}^{N} R_{i}\left(\int_{\Gamma_{i}} \mathbf{u}_{0} \cdot \mathbf{n}\right)^{2}
$$

is well-defined over $\left[0, t^{\star}\right]$. By a lemma of comparison for differential inequalities (see e.g. [21]), we deduce from (25) that $\mathbf{u}_{n}$ is well-defined as the unique solution to (23) over $\left[0, t^{\star}\right]$ (in particular, $\left.t^{\star}<t_{n}\right)$, and that

$$
\Phi_{n}(t)=\mu\left\|\nabla \mathbf{u}_{n}\right\|_{L^{2}(\Omega)}^{2}+\sum_{i=1}^{N} R_{i}\left(\int_{\Gamma_{i}} \mathbf{u}_{n} \cdot \mathbf{n}\right)^{2}+k \int_{0}^{t}\left\|\nabla \mathbf{u}_{n}\right\|_{L^{2}(\Omega)}^{2} \leq F(t), \quad \forall t \in\left[0, t^{\star}\right] .
$$

Besides, by integrating $(25)$ over $\left(0, t^{\star}\right)$, we get

$$
\int_{0}^{t^{\star}}\left\|A \mathbf{u}_{n}\right\|_{0}^{2} \leq C<\infty
$$

We thus deduce that $\mathbf{u}_{n}$ is uniformly bounded in $L^{\infty}\left(0, t^{*} ; V\right) \cap L^{2}\left(0, t^{*} ; D(A)\right)$. Thanks to these estimates we can easily deduce a uniform bound for $\int_{0}^{t}\left\|\frac{\partial \mathbf{u}_{n}}{\partial \tau}\right\|_{L^{2}(\Omega)}^{2} d \tau$, by choosing $\mathbf{v}=\frac{\partial \mathbf{u}_{n}}{\partial t}$ in (18). Indeed

$$
\begin{aligned}
\left\|\frac{\partial \mathbf{u}_{n}}{\partial t}\right\|_{0}^{2}= & -\rho \int_{\Omega}\left(\mathbf{u}_{n} \cdot \nabla \mathbf{u}_{n}\right) \frac{\partial \mathbf{u}_{n}}{\partial t}-\left(A \mathbf{u}_{n}, \frac{\partial \mathbf{u}_{n}}{\partial t}\right) \\
& -k\left(\int_{0}^{t} \int_{\Gamma_{0}} \mathbf{u}_{n} \cdot \mathbf{n}\right)\left(\int_{\Gamma_{0}} \frac{\partial \mathbf{u}_{n}}{\partial t} \cdot \mathbf{n}\right)+\ell\left(\frac{\partial \mathbf{u}_{n}}{\partial t}\right)
\end{aligned}
$$


and thus, using Lemma 2.6, the equivalence of the $L^{2}$ norm and $\|\cdot\|_{0}$ on $H$, estimates (27) and (28), and Corollary 2.9,

$$
\begin{aligned}
\left\|\frac{\partial \mathbf{u}_{n}}{\partial t}\right\|_{L^{2}(\Omega)}^{2} & \leq C_{T}\left(\left\|\mathbf{u}_{n}\right\|_{L^{\infty}(\Omega)}\left\|\nabla \mathbf{u}_{n}\right\|_{L^{2}(\Omega)}+\left\|A \mathbf{u}_{n}\right\|_{L^{2}(\Omega)}+\left|P_{0}\right|+\frac{\left|f_{\text {ext }}\right|}{S}+\frac{k\left|x_{0}\right|}{S}\right)\left\|\frac{\partial \mathbf{u}_{n}}{\partial t}\right\|_{L^{2}(\Omega)}, \\
\left\|\frac{\partial \mathbf{u}_{n}}{\partial t}\right\|_{L^{2}(\Omega)} & \leq C_{T}\left(\left\|\nabla \mathbf{u}_{n}\right\|_{L^{2}(\Omega)}^{1+\theta}\left\|A \mathbf{u}_{n}\right\|_{L^{2}(\Omega)}^{1-\theta}+\left\|A \mathbf{u}_{n}\right\|_{L^{2}(\Omega)}+\left|P_{0}\right|+\frac{\left|f_{\text {ext }}\right|}{S}+\frac{k\left|x_{0}\right|}{S}\right) .
\end{aligned}
$$

The previous bounds, together with (22), thus imply that $\frac{\partial \mathbf{u}_{n}}{\partial t}$ is uniformly bounded in $L^{2}\left(0, t^{*} ; L^{2}(\Omega)\right)$. Passage to the limit

Consequently there exists a subsequence of $\left(\mathbf{u}_{n}\right)$, also denoted $\left(\mathbf{u}_{n}\right)$, such that

$$
\left\{\begin{array}{l}
\mathbf{u}_{n} \rightarrow \mathbf{u} \text { weakly }{ }^{*} \text { in } L^{\infty}\left(0, t^{*} ; V\right) \\
\text { and } \\
\frac{\partial \mathbf{u}_{n}}{\partial t} \rightarrow \frac{\partial \mathbf{u}}{\partial t} \text { weakly in } L^{2}\left(0, t^{*} ; L^{2}(\Omega)\right) .
\end{array}\right.
$$

Futhermore thanks to Aubin's compactess lemma (see [24], p. 57) we have, up to a subsequence (still denoted $\left(\mathbf{u}_{n}\right)$ ),

$$
\mathbf{u}_{n} \underset{n \rightarrow+\infty}{\longrightarrow} \mathbf{u} \text { in } L^{2}\left(0, t^{*} ; L^{2}(\Omega)\right) .
$$

It enables us to pass to the limit in the discrete problem and to obtain the existence, locally in time, of at least one solution $\mathbf{u}$ of (18).

Global existence. Here we consider the special case where the spring stiffness $k$ is equal to zero. Moreover we assume that the data $P_{0}, f_{\text {ext }}$ are in $L^{\infty}(0, T)$. Once again, by taking $A \mathbf{u}_{n}$ as a test function in the discrete problem yields

$$
\begin{aligned}
& \frac{\mu}{2} \frac{d}{d t}\left\|\nabla \mathbf{u}_{n}\right\|_{L^{2}(\Omega)}^{2}+\frac{1}{2} \sum_{i=1}^{N} R_{i} \frac{d}{d t}\left(\int_{\Gamma_{i}} \mathbf{u}_{n} \cdot \mathbf{n}\right)^{2}+\left\|A \mathbf{u}_{n}\right\|_{0}^{2} \leq \\
& \rho\left\|\mathbf{u}_{n}\right\|_{L^{\infty}(\Omega)}\left\|\nabla \mathbf{u}_{n}\right\|_{L^{2}(\Omega)}\left\|A \mathbf{u}_{n}\right\|_{L^{2}(\Omega)}+C\left(\left|P_{0}\right|+\frac{\left|f_{\text {ext }}\right|}{S}\right)\left\|A \mathbf{u}_{n}\right\|_{L^{2}(\Omega)} .
\end{aligned}
$$

By Corollary 2.9 we have

$$
\left\|\mathbf{u}_{n}\right\|_{L^{\infty}(\Omega)} \leq C\left\|A \mathbf{u}_{n}\right\|_{0}
$$

thus, using Young inequality and the equivalence of the $L^{2}$ norm and $\|\cdot\|_{0}$ on $H$,

$$
\begin{aligned}
\frac{\mu}{2} \frac{d}{d t}\left\|\nabla \mathbf{u}_{n}\right\|_{L^{2}(\Omega)}^{2}+ & \frac{1}{2} \sum_{i=1}^{N} R_{i} \frac{d}{d t}\left(\int_{\Gamma_{i}} \mathbf{u}_{n} \cdot \mathbf{n}\right)^{2}+\left\|A \mathbf{u}_{n}\right\|_{0}^{2} \\
& \leq C\left\|A \mathbf{u}_{n}\right\|_{0}^{2}\left\|\nabla \mathbf{u}_{n}\right\|_{L^{2}(\Omega)}+C\left(\left|P_{0}\right|^{2}+\frac{\left|f_{\text {ext }}\right|^{2}}{S^{2}}\right)+\frac{1}{2}\left\|A \mathbf{u}_{n}\right\|_{0}^{2},
\end{aligned}
$$


and consequently

$$
\mu \frac{d}{d t}\left\|\nabla \mathbf{u}_{n}\right\|_{L^{2}(\Omega)}^{2}+\sum_{i=1}^{N} R_{i} \frac{d}{d t}\left(\int_{\Gamma_{i}} \mathbf{u}_{n} \cdot \mathbf{n}\right)^{2}+\left(1-C\left\|\nabla \mathbf{u}_{n}\right\|_{L^{2}(\Omega)}\right)\left\|A \mathbf{u}_{n}\right\|_{0}^{2} \leq C\left(\left|P_{0}\right|^{2}+\frac{\left|f_{\text {ext }}\right|^{2}}{S^{2}}\right) .
$$

Thanks to the definition of $A$ we have $\left\|\nabla \mathbf{u}_{n}\right\|_{L^{2}(\Omega)} \leq C\left\|A \mathbf{u}_{n}\right\|_{0}$ and since $\left|\int_{\Gamma_{i}} \mathbf{v} \cdot \mathbf{n}\right| \leq C\|\nabla \mathbf{v}\|_{L^{2}(\Omega)}$ for any $\mathbf{v} \in H^{1}(\Omega)$, we then obtain an inequality of the type

$$
\frac{d}{d t} \Phi_{n}+B_{1}\left(1-B_{2} \sqrt{\Phi}_{n}\right) \Phi_{n} \leq C\left(\left|P_{0}\right|^{2}+\frac{\left|f_{e x t}\right|^{2}}{S^{2}}\right)
$$

which is valid provided $1-B_{2} \sqrt{\Phi}_{n}>0$ and where

$$
\Phi_{n}=\mu\left\|\nabla \mathbf{u}_{n}\right\|_{L^{2}(\Omega)}^{2}+\sum_{i=1}^{N} R_{i}\left(\int_{\Gamma_{i}} \mathbf{u}_{n} \cdot \mathbf{n}\right)^{2}
$$

and $B_{1}, B_{2}$ are positive constants. Consequently, assuming

$$
C\left(\left|P_{0}\right|^{2}+\frac{\left|f_{\text {ext }}\right|^{2}}{S^{2}}\right) \leq \frac{B_{1}}{8 B_{2}^{2}} \text { and } \mu\left\|\nabla \mathbf{u}_{0}\right\|_{L^{2}(\Omega)}^{2}+\sum_{i=1}^{N} R_{i}\left(\int_{\Gamma_{i}} \mathbf{u}_{0} \cdot \mathbf{n}\right)^{2} \leq \frac{1}{2 B_{2}}
$$

the latter inequality implying that $\sqrt{\Phi_{n}(0)} \leq \frac{1}{2 B_{2}}$ (see $(26)$ ), we can check that $\Phi_{n}$ is defined over $[0,+\infty)$, with

$$
\sqrt{\Phi_{n}(t)} \leq \frac{1}{2 B_{2}} \quad \forall t
$$

Under these assumptions, the solution is defined globally in time.

Remark 2.12 Here we assumed that $k=0$ because we are not able to control the displacement of the spring. Nevertheless, if we assume a priori that the spring displacement is bounded by a given (small enough) constant then one could obtain the same global existence result in the case of a non zero spring stiffness. On the other hand, global existence of weak solutions can be established without any assumptions on the data (including the stiffness $k$ ) if neglected the non linear convective terms of the Navier-Stokes equations. The difficulty here lies in the fact that the nonlinear system we consider (Navier-Stokes + spring-mass) is capable of storing energy in a form which is not counterbalanced by any dissipation phenomenon (potential energy of the spring).

Uniqueness. Let us consider two solutions $\mathbf{u}_{1}, \mathbf{u}_{2}$ of (18):

$$
\left(\frac{\partial \mathbf{u}_{i}}{\partial t}, \mathbf{v}\right)_{0}+a_{1}\left(\mathbf{u}_{i}, \mathbf{v}\right)+a_{2}\left(\int_{0}^{t} \mathbf{u}_{i}, \mathbf{v}\right)+b\left(\mathbf{u}_{i}, \mathbf{u}_{i}, \mathbf{v}\right)=\ell(\mathbf{v}) \quad \forall \mathbf{v} \in V, i=1,2,
$$


associated to the same initial data $\mathbf{u}_{0}$. Setting $\mathbf{w}=\mathbf{u}_{1}-\mathbf{u}_{2}$, substracting the two previous equations and taking $\mathbf{v}=\mathbf{w}$ as a test function, we obtain

$$
\begin{aligned}
& \frac{1}{2} \frac{d}{d t}\|\mathbf{w}\|_{0}^{2}+\frac{k}{2 S^{2}} \frac{d}{d t}\left(\int_{\Gamma_{0}} \mathbf{w} \cdot \mathbf{n}\right)^{2}+\mu\|\nabla \mathbf{w}\|_{L^{2}(\Omega)}^{2}+\sum_{i=1}^{N} R_{i}\left(\int_{\Gamma_{i}} \mathbf{w} \cdot \mathbf{n}\right)^{2} \\
= & b\left(\mathbf{u}_{1}, \mathbf{u}_{1}, \mathbf{w}\right)-b\left(\mathbf{u}_{2}, \mathbf{u}_{2}, \mathbf{w}\right) .
\end{aligned}
$$

We have

$$
b\left(\mathbf{u}_{1}, \mathbf{u}_{1}, \mathbf{w}\right)-b\left(\mathbf{u}_{2}, \mathbf{u}_{2}, \mathbf{w}\right)=b\left(\mathbf{u}_{2}, \mathbf{w}, \mathbf{w}\right)+b\left(\mathbf{w}, \mathbf{u}_{2}, \mathbf{w}\right)+b(\mathbf{w}, \mathbf{w}, \mathbf{w}),
$$

and

$$
\left|b\left(\mathbf{u}_{2}, \mathbf{w}, \mathbf{w}\right)\right| \leq\left\|\mathbf{u}_{2}\right\|_{L^{\infty}(\Omega)}\|\nabla \mathbf{w}\|_{L^{2}(\Omega)}\|\mathbf{w}\|_{L^{2}(\Omega)} .
$$

Besides, thanks to Hölder inequality,

$$
\left|b\left(\mathbf{w}, \mathbf{u}_{2}, \mathbf{w}\right)\right| \leq\|\mathbf{w}\|_{L^{6}(\Omega)}\left\|\nabla \mathbf{u}_{2}\right\|_{L^{2}(\Omega)}\|\mathbf{w}\|_{L^{3}(\Omega)}
$$

and

$$
|b(\mathbf{w}, \mathbf{w}, \mathbf{w})| \leq\|\mathbf{w}\|_{L^{6}(\Omega)}\|\nabla \mathbf{w}\|_{L^{2}(\Omega)}\|\mathbf{w}\|_{L^{3}(\Omega)} .
$$

Now Sobolev injections for $d=2,3$ lead to

$$
\|\mathbf{v}\|_{L^{6}(\Omega)} \leq C\|\nabla \mathbf{v}\|_{L^{2}(\Omega)}
$$

and

$$
\|\mathbf{v}\|_{L^{3}(\Omega)} \leq C\|\mathbf{v}\|_{L^{2}(\Omega)}^{\frac{1}{2}}\|\nabla \mathbf{v}\|_{L^{2}(\Omega)}^{\frac{1}{2}} .
$$

These estimates, along with Young and Poincaré's inequalities, imply

$$
\begin{aligned}
\frac{1}{2} \frac{d}{d t}\|\mathbf{w}\|_{0}^{2}+\frac{k}{2 S^{2}} \frac{d}{d t}\left(\int_{\Gamma_{0}} \mathbf{w} \cdot \mathbf{n}\right)^{2}+\mu\|\nabla \mathbf{w}\|_{L^{2}(\Omega)}^{2} & \leq C\left(\left\|\mathbf{u}_{2}\right\|_{L^{\infty}(\Omega)}\|\mathbf{w}\|_{L^{2}(\Omega)}\right)^{2}+\frac{\mu}{4}\|\nabla \mathbf{w}\|_{L^{2}(\Omega)}^{2} \\
& +C\left\|\nabla \mathbf{u}_{2}\right\|_{L^{2}(\Omega)}^{4}\|\mathbf{w}\|_{L^{2}(\Omega)}^{2}+\frac{\mu}{4}\|\nabla \mathbf{w}\|_{L^{2}(\Omega)}^{2} \\
& +C\|\mathbf{w}\|_{L^{2}(\Omega)}^{\frac{1}{2}}\|\nabla \mathbf{w}\|_{L^{2}(\Omega)}^{\frac{5}{2}} .
\end{aligned}
$$

Finally

$$
\begin{aligned}
& \frac{\rho}{2} \frac{d}{d t}\|\mathbf{w}\|_{0}^{2}+\frac{k}{S^{2}} \frac{d}{d t}\left(\int_{\Gamma_{0}} \mathbf{w} \cdot \mathbf{n}\right)^{2}+\left(\mu-C\|\mathbf{w}\|_{L^{2}(\Omega)}^{\frac{1}{2}}\|\nabla \mathbf{w}\|_{L^{2}(\Omega)}^{\frac{1}{2}}\right)\|\nabla \mathbf{w}\|_{L^{2}(\Omega)}^{2} \\
\leq & C\left(\left\|\mathbf{u}_{2}\right\|_{L^{\infty}(\Omega)}^{2}+\left\|\nabla \mathbf{u}_{2}\right\|_{L^{2}(\Omega)}^{4}\right)\|\mathbf{w}\|_{L^{2}(\Omega)}^{2}
\end{aligned}
$$

and thus on a time interval on which $\|\mathbf{w}\|_{L^{2}(\Omega)}^{\frac{1}{2}}\|\nabla \mathbf{w}\|_{L^{2}(\Omega)}^{\frac{1}{2}} \leq \frac{\mu}{C}$, we have, since the $L^{2}$ norm and $\|\cdot\|_{0}$ are equivalent on $H$,

$$
\frac{d}{d t}\|\mathbf{w}\|_{0}^{2}+\frac{k}{S^{2}} \frac{d}{d t}\left(\int_{\Gamma_{0}} \mathbf{w} \cdot \mathbf{n}\right)^{2} \leq C\left(\left\|\mathbf{u}_{2}\right\|_{L^{\infty}(\Omega)}^{2}+\left\|\nabla \mathbf{u}_{2}\right\|_{L^{2}(\Omega)}^{4}\right)\|\mathbf{w}\|_{0}^{2} .
$$


Taking into account that $\mathbf{u}_{2}$ is bounded in $L^{\infty}(0, T ; V) \cap L^{2}(0, T ; D(A))$ and the fact that $D(A) \subset$ $L^{\infty}(\Omega)$ (see Corollary 2.9), Gronwall lemma implies that $\mathbf{w} \equiv 0$ since $\mathbf{w}(0)=0$. Note that the same type of estimates could be used to prove that the solution depends continuously on the data. In particular, if $\left\|\mathbf{u}_{2}\right\|_{L^{\infty}(\Omega)}^{2}+\left\|\nabla \mathbf{u}_{2}\right\|_{L^{2}(\Omega)}^{4}$ is small enough, using (32) then any small perturbation $\mathbf{w}$ of $\mathbf{u}_{2}$ decreases exponentially.

Remark 2.13 The operator A which we use to establish the existence result is not the classical Stokes operator: it takes into account the dissipative terms associated to the resistances $R_{i}$. If we had chosen not to take them into account, the existence result, even locally in time, would have required some control of these resistances (see [33] where a similar proof is proposed).

\section{$3 \quad$ Numerical method}

We present the numerical strategy we adopted, which leads to an implicit implementation of the dissipative boundary conditions (see [26]).

Note that most of the numerical studies in realistic airways geometries we are aware of (see [9], [11], [12], [14]) use commercial softwares, with Neumann or Dirichlet boundary conditions at the inlet and outlets, to perform their flow simulations. Nervertheless most "general purpose" softwares are not designed to take into account easily natural dissipative boundary conditions, nor the coupling with the spring model.

\subsection{Time discretization}

Theoretical results have been obtained in the space of divergence free functions. For the sake of efficiency, the numerical approach relies on a mixed formulation (non divergence free test functions are used, and the divergence free constraint is expressed in a weak form). Consequently, the formulation is obtained by multiplying (10) with general test functions $\mathbf{v} \in H^{1}(\Omega)$ that vanish on $\Gamma_{\ell}$. Thus, we obtain the variational formulation (11), with the unknown pressure $\bar{p}=p-P_{a}$, along wih the constraint equation $\int_{\Omega} q \nabla \cdot \mathbf{u}=0$, for all $q \in L^{2}(\Omega)$, that is

$$
\left\{\begin{array}{l}
\rho \int_{\Omega} \partial_{t} \mathbf{u} \cdot \mathbf{v}+\rho \int_{\Omega}(\mathbf{u} \cdot \nabla) \mathbf{u} \cdot \mathbf{v}+\mu \int_{\Omega} \nabla \mathbf{u}: \nabla \mathbf{v}+\sum_{i=1}^{N} R_{i}\left(\int_{\Gamma_{i}} \mathbf{u} \cdot \mathbf{n}\right)\left(\int_{\Gamma_{i}} \mathbf{v} \cdot \mathbf{n}\right) \\
+\frac{m}{S^{2}}\left(\int_{\Gamma_{0}} \partial_{t} \mathbf{u} \cdot \mathbf{n}\right)\left(\int_{\Gamma_{0}} \mathbf{v} \cdot \mathbf{n}\right)+\frac{k}{S^{2}}\left(\int_{0}^{t} \int_{\Gamma_{0}} \mathbf{u} \cdot \mathbf{n}\right)\left(\int_{\Gamma_{0}} \mathbf{v} \cdot \mathbf{n}\right) \\
-\int_{\Omega} \bar{p} \nabla \cdot \mathbf{v} \\
=-P_{0} \int_{\Gamma_{0}} \mathbf{v} \cdot \mathbf{n}-\frac{f_{\text {ext }}}{S} \int_{\Gamma_{0}} \mathbf{v} \cdot \mathbf{n}-\frac{k}{S^{2}} S x_{0}\left(\int_{\Gamma_{0}} \mathbf{v} \cdot \mathbf{n}\right) \quad \forall \mathbf{v} \in H^{1}(\Omega), \mathbf{v}=0 \text { on } \Gamma_{\ell} \\
\int_{\Omega} q \nabla \cdot \mathbf{u}=0 \quad \forall q \in L^{2}(\Omega) .
\end{array}\right.
$$

Let $\delta t>0$ be the time step, and $t^{n}=n \delta t, n \in \mathbb{N}$. We denote by $\mathbf{u}^{n}$ the approximated solution at time $t^{n}$. Using a semi-implicit scheme for the non-linear term in the variational formulation, and the 
following approximation for the time-integral term $\int_{0}^{t^{n}} \int_{\Gamma_{0}} \mathbf{u} \cdot \mathbf{n}$ in (33),

$$
\int_{0}^{t^{n}} \int_{\Gamma_{0}} \mathbf{u} \cdot \mathbf{n} \approx \sum_{j=0}^{n}\left(\delta t \int_{\Gamma_{0}} \mathbf{u}^{j} \cdot \mathbf{n}\right)
$$

the time discretization reads as follows

$$
\left\{\begin{array}{l}
\frac{\rho}{\delta t} \int_{\Omega} \mathbf{u}^{n} \cdot \mathbf{v}+\rho \int_{\Omega}\left(\mathbf{u}^{n} \cdot \nabla\right) \mathbf{u}^{n-1} \cdot \mathbf{v}+\mu \int_{\Omega} \nabla \mathbf{u}^{n}: \nabla \mathbf{v}+\sum_{i=1}^{N} R_{i}\left(\int_{\Gamma_{i}} \mathbf{u}^{n} \cdot \mathbf{n}\right)\left(\int_{\Gamma_{i}} \mathbf{v} \cdot \mathbf{n}\right) \\
+\left(\frac{m}{S^{2} \delta t}+\frac{k \delta t}{S^{2}}\right)\left(\int_{\Gamma_{0}} \mathbf{u}^{n} \cdot \mathbf{n}\right)\left(\int_{\Gamma_{0}} \mathbf{v} \cdot \mathbf{n}\right)-\int_{\Omega} \bar{p}^{n} \nabla \cdot \mathbf{v} \\
=\left[-P_{0}-\frac{f_{e x t}^{n}}{S}+\frac{k}{S} x_{0}-\frac{k}{S^{2}}\left(\sum_{j=0}^{n-1} \delta t \int_{\Gamma_{0}} \mathbf{u}^{j} \cdot \mathbf{n}\right)\right]\left(\int_{\Gamma_{0}} \mathbf{v} \cdot \mathbf{n}\right)+ \\
\frac{m}{S^{2} \delta t}\left(\int_{\Gamma_{0}} \mathbf{u}^{n-1} \cdot \mathbf{n}\right)\left(\int_{\Gamma_{0}} \mathbf{v} \cdot \mathbf{n}\right)+\frac{\rho}{\delta t} \int_{\Omega} \mathbf{u}^{n-1} \cdot \mathbf{v} \quad \forall \mathbf{v} \in H^{1}(\Omega), \mathbf{v}=0 \text { on } \Gamma_{\ell} \\
\int_{\Omega} q \nabla \cdot \mathbf{u}^{n}=0 \quad \forall q \in L^{2}(\Omega) .
\end{array}\right.
$$

Using the bilinear forms $a_{1}(\cdot, \cdot)$ and $a_{2}(\cdot, \cdot)$ and the trilinear form $b(\cdot, \cdot, \cdot)$ introduced in Section 2.1, and the bilinear form $c(\cdot, \cdot)$ defined by

$$
c(\mathbf{u}, p)=-\int_{\Omega} p \nabla \cdot \mathbf{u}
$$

the semi-discretized variational formulation can be written as follows

$$
\left\{\begin{array}{l}
\frac{1}{\delta t}\left(\mathbf{u}^{n}, \mathbf{v}\right)_{0}+a_{1}\left(\mathbf{u}^{n}, \mathbf{v}\right)+\delta t a_{2}\left(\mathbf{u}^{n}, \mathbf{v}\right) \\
+b\left(\mathbf{u}^{n}, \mathbf{u}^{n-1}, \mathbf{v}\right)+c\left(\mathbf{v}, \bar{p}^{n}\right)=\ell^{n}(\mathbf{v}) \quad \forall \mathbf{v} \in H^{1}(\Omega), \mathbf{v}=0 \text { on } \Gamma_{\ell} \\
c\left(\mathbf{u}^{n}, q\right)=0 \quad \forall q \in L^{2}(\Omega) .
\end{array}\right.
$$

where the right-hand side $\ell^{n}(\mathbf{v})$ is given by

$$
\ell^{n}(\mathbf{v})=\left[-P_{0}-\frac{f_{e x t}^{n}}{S}+\frac{k}{S} x_{0}-\frac{k}{S^{2}}\left(\sum_{j=0}^{n-1} \delta t \int_{\Gamma_{0}} \mathbf{u}^{j} \cdot \mathbf{n}\right)\right]\left(\int_{\Gamma_{0}} \mathbf{v} \cdot \mathbf{n}\right)+\frac{1}{\delta t}\left(\mathbf{u}^{n-1}, \mathbf{v}\right)_{0} .
$$

Remark 3.1 The discretization (34) of the integral term $\int_{0}^{t^{n}} \int_{\Gamma_{0}} \mathbf{u} \cdot \mathbf{n}$ can be associated to an implicit discretization of the constraint equation $S \dot{x}=-\int_{\Gamma_{0}} \mathbf{u} \cdot \mathbf{n}$ (see Eq. (7)). Indeed, using backward difference, we have

$$
x^{n}=x^{n-1}-\frac{\delta t}{S} \int_{\Gamma_{0}} \mathbf{u}^{n} \cdot \mathbf{n},
$$


so, by downward recursion to initial position $x^{0}$,

$$
x^{n}=x^{0}-\frac{\delta t}{S} \sum_{j=0}^{n} \int_{\Gamma_{0}} \mathbf{u}^{j} \cdot \mathbf{n},
$$

so that the mass position at time $t^{n}$ is implicitly calculated and can be determined using (37) or (38) after the resolution of the modified Navier-Stokes system (36).

In the same way, part of the right-hand side of the mixed variational formulation (36) corresponds to the following time discretization of the mass-spring differential equation (4)-(5), coupled with the constraint equation (6) or (7), using an implicit central difference scheme

$$
P_{a}^{n}=\frac{1}{S}\left(m \frac{x^{n}-2 x^{n-1}+x^{n-2}}{\delta t^{2}}+k x^{n}-f_{e x t}^{n}\right)
$$

and using (37) and (38),

$$
P_{a}^{n}=\frac{1}{S}\left(-\frac{m}{\delta t}\left(\int_{\Gamma_{0}} \mathbf{u}^{n} \cdot \mathbf{n}-\int_{\Gamma_{0}} \mathbf{u}^{n-1} \cdot \mathbf{n}\right)+k\left(x^{0}-\frac{\delta t}{S} \sum_{j=0}^{n} \int_{\Gamma_{0}} \mathbf{u}^{j} \cdot \mathbf{n}\right)-f_{e x t}^{n}\right) .
$$

Remark 3.2 Note that another-equivalent-discretization strategy consists in consider the variational formulation obtained by using (8) as the starting system, instead of the system (10). In this approach, the surface integral terms on $\Gamma_{0}$ would be written in terms of the sum of surface integrals on $\Gamma_{i}$, that is in the semi-discretized variational formulation we would deal with a quadratic term of the form

$$
\left(\frac{m}{S^{2} \delta t}+\frac{k \delta t}{S^{2}}\right)\left(\sum_{i=1}^{N} \int_{\Gamma_{i}} \mathbf{u}^{n} \cdot n\right)\left(\sum_{i=1}^{N} \int_{\Gamma_{i}} \mathbf{v} \cdot n\right)
$$

Hence, in a finite element framework, this would imply that all the degrees of freedom belonging to the outlet boundaries $\Gamma_{i}, i=1, \ldots, N$, would be coupled. The two formulations are equivalent as far as discrete solutions are concerned. Yet, the resulting matrices differ in the way degrees of freedom are coupled, and numerical tests suggest that the system resulting from formulation (35) is better conditionned.

\subsection{Space discretization}

The solver uses a $P 1 / P 1$ stabilized finite element method (FEM) for the space discretization of NavierStokes systems. The linear systems obtained are then solved using a preconditioned GMRES iterative routine.

As seen before, the dissipative conditions coming from the resistance and the mass-spring timediscretization modify the bilinear forms in the variational formulation and, therefore, they would modify the FEM matrix associated to the velocity degrees of freedom. However, from the variational formulation we see that these modifications can be seen as an additive perturbation of the original FEM matrix,

$$
A=A^{\Omega}+A^{r e s} .
$$


where $A^{\Omega}$ stands for the standard FEM matrix over $\Omega$, and $A^{\text {res }}$ stands for the matrix associated to resistances.

The matrix $A^{\text {res }}$ is not assembled : matrix-vector products involving $A^{\text {res }}$ are performed as tensor operations based on a vector whose entries depend on $\int_{\Gamma_{j}} \phi_{i} \cdot \mathbf{n}$ (where $\phi_{i}$ is a FEM velocity basis function), the resistance values $R_{j}, j=1, \ldots, N$, and the modified resistance value $\tilde{R}_{0}=R_{0}+$ $S^{-2}\left(m \delta t^{-1}+k \delta t\right)$ (for the entries associated to nodes on $\Gamma_{0}$ ).

Remark 3.3 When considering only the Navier-Stokes system with natural dissipative boundary conditions (3) then one could impose these conditions explicitly. In this case the FEM matrice of the system is $A^{\Omega}$. Nervetheless for large resistance value $R_{i}$ this may be unstable, as suggested by the sufficient existence condition that one will obtain by omitting the resistive part in the definition of the bilinear form of the system (see Remark 2.13).

Remark 3.4 If the second variational formulation were used (see Remark 3.2), then the matrix product $A^{\text {res } x}$ would be more complicated, since all the outlets' degrees of freedom would be coupled. Moreover each entry associated to an outlet's degree of freedom would depend on the coefficient $S^{-2}\left(m \delta t^{-1}+k \delta t\right)$ coming from the spring-mass system time discretization.

\section{Note on the preconditioner}

As the preconditioner for the modified linear system we use the incomplete LU (ILU) preconditioner associated to the original FEM matrices which demonstrates to be sufficient for the physiological numerical tests we performed. Nevertheless for large values of the subtrees resistance this preconditioner may not be appropriated.

\section{Numerical results}

In this section we present $3 D$ numerical results of the coupled Navier-Stokes/mass-spring system, performed with an academic code developed at INRIA-Rocquencourt which has implemented the natural dissipative boundary conditions in an implicit way (see [26]). We illustrate how this approach makes it possible to investigate the effect of a modification of some resistances in the condensed (distal) part onto the overall flow in the upper (proximal) part of the tree. We will also test the effect of a modification of the spring stiffness. The goal of this section is to illustrate the feasibility from a numerical point of view of the model using a realistic geometry and realistic data. The precise physiological exploitation of the multiscale system will be the object of a forthcoming paper.

\subsection{Methodology, data}

We have performed numerical calculations of the coupled Navier-Stokes/mass-spring system in a $3 D$ reconstructed bronchial tree (until $6 t h-7 t h$ generation). The tetrahedral meshes (see Fig. 2) were constructed using INRIA GHS3D mesh generator [16]. The original $3 D$ surface geometry is the same used in $[14,13]$, and it was reconstructed, from CRT medical images, using a marching cube-base adaptive approach (see [14] for more details) . 


\section{Mesh size}

We use a tetrahedral mesh composed of $N_{\text {nodes }}=31288$ nodes, $N_{\text {tetra }}=131562$ tetrahedra and $N_{\text {triang }}=33950$ surface triangles.

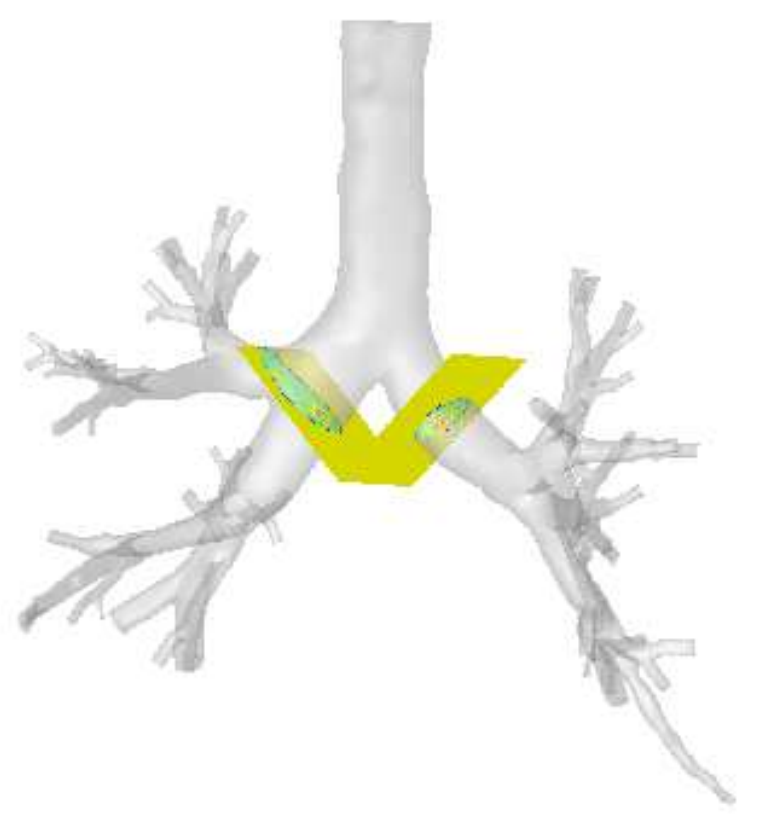

Figure 2: Reconstructed bronchial tree. Cut planes (yellow) after first bifurcation.

\section{Resistance values}

The resistance values were estimated using anatomical data from [40], assuming that each outlet $\Gamma_{i}$ is connected to a dyadic subtree which is assumed to follow a geometric decrease of the pipe sizes, and therefore a geometric increase of individual pipe resistances. As the outlets correspond to different generations $\left(j_{0}=4,5,6,7\right.$, and 8$)$, the corresponding resistances are computed as partial sums of the type

$$
R=\sum_{j=j_{0}}^{17} \frac{r_{j}}{2^{j-j_{0}+1}}, \quad r_{j}=\frac{128 \mu}{\pi} \frac{l_{j}}{d_{j}^{4}},
$$

where $l_{j}$ and $d_{j}$ stand for the bronchus' length and diameter, respectively. Note that a dissipative boundary condition is prescribed at inlet $\Gamma_{0}$, to account for the resistance of the upper part of the respiratory tract (nose, pharynx, and larynx). The corresponding resistance value is taken from [4, 25]. 
The table below presents the resistance values (in $\mathrm{g} \cdot \mathrm{cm}^{-4} \cdot \mathrm{s}^{-1}$ ) for the inlet and for the 58 outlets (the value depends on the generation $j_{0}$ only).

\begin{tabular}{|c|c|}
\hline generation $\left(j_{0}\right)$ & $R$ \\
\hline 0 & $1.12 \mathrm{E}+00$ \\
4 & $2.24 \mathrm{E}+00$ \\
5 & $3.89 \mathrm{E}+00$ \\
6 & $6.59 \mathrm{E}+00$ \\
7 & $1.10 \mathrm{E}+01$ \\
8 & $1.70 \mathrm{E}+01$ \\
\hline
\end{tabular}

\section{Fluid and spring parameters}

The fluid is supposed to be air saturated in water vapor, and its dynamic viscosity and density are given by

$$
\mu=1.98 \times 10^{-4} \mathrm{~g} \cdot \mathrm{cm}^{-1} \cdot \mathrm{s}^{-1}, \quad \rho=1.11 \times 10^{-3} \mathrm{~g} \cdot \mathrm{cm}^{-3}
$$

so that the kinematic viscosity is given by $\nu=\mu / \rho=0.18 \mathrm{~cm}^{2} \cdot \mathrm{s}^{-1}$. For the mass-spring system, we have used the same values as in [25]:

$$
m=300 \mathrm{~g}, \quad S=100 \mathrm{~cm}^{2}, \quad k=3.63 \times 10^{3} \mathrm{dyn} \cdot \mathrm{cm}^{-1},
$$

\subsection{Results}

We present the numerical results obtained in the case of normal respiration simulations and in the case of forced maneuvers simulations. In the case of normal respiration simulations we apply a periodic smoothed pulse force (of period $T=4 s$ ) with $f_{\max }=1.5 \times 10^{5} d y n$ and $f_{\min }=0$. Figure 3 shows the mass-spring displacement $x$ (in $\mathrm{cm}$ ) versus time $t$ (in $s$ ) and air flow $Q$ (in $\mathrm{cm}^{3} \cdot \mathrm{s}^{-1}$ ) through boundary $\Gamma_{0}$ versus time $t$. Note that $x_{\max } \approx 4 \mathrm{~cm}$, therefore the tidal volume $V_{T}$ is approximately
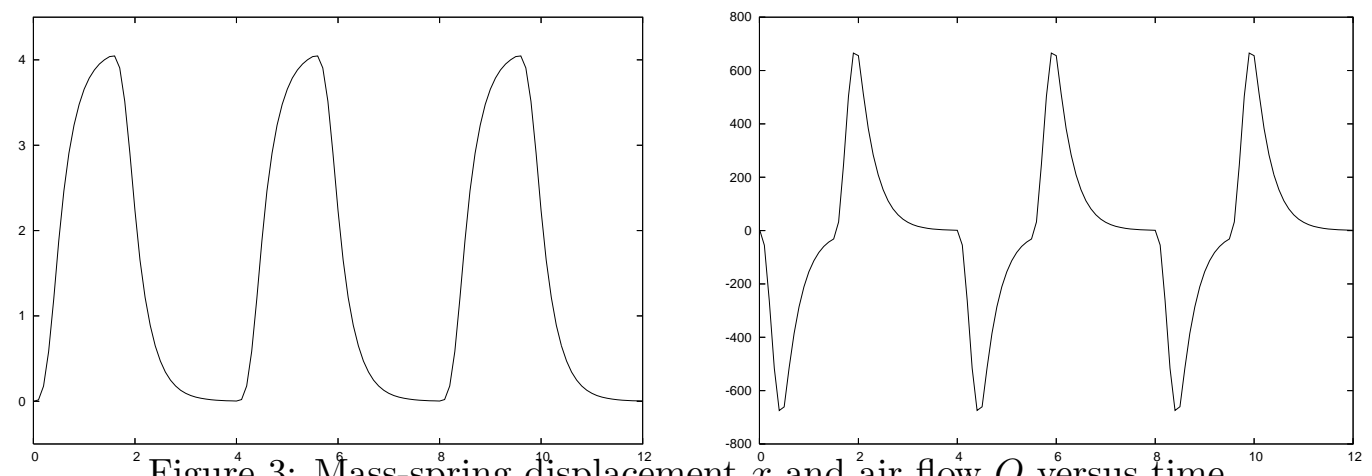

${ }^{2}$ Figure 3: Mass-spring displacement $\stackrel{4}{x}$ and ${ }^{12}$ air flow $Q$ versus time.

equal to $400 \mathrm{~cm}^{3}$, since $S=100 \mathrm{~cm}^{2}$, which is the average value for an healthy adult. 

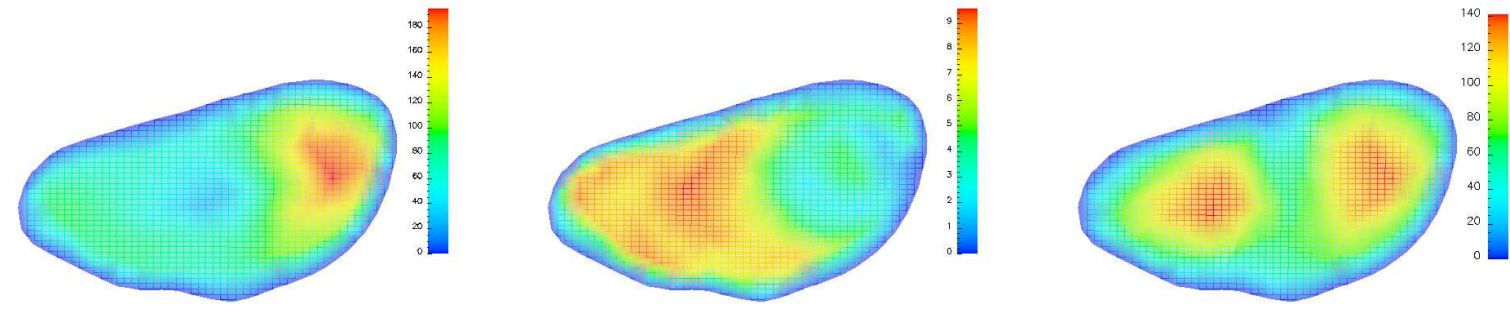

Figure 4: Isovalues of the velocity vector field norm on cut plane on left first bifurcation, at peak inspiration $(t=0.4 s$, left $)$, transition $(t=1.6 s$, middle $)$, peak expiration $(t=1.9 \mathrm{~s}$, right $)$
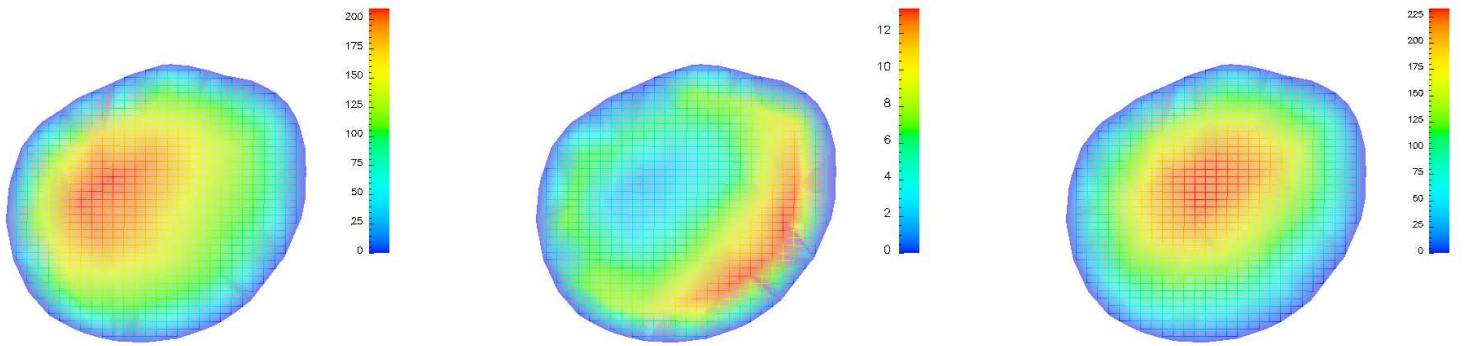

Figure 5: Isovalues of the velocity modulus on cut plane on right first bifurcation. at peak inspiration $(t=0.4$, left $)$, transition $(t=1.6$, middle), peak expiration $(t=1.9$, right $)$

Figures 4 and 5 show the isovalues of the velocity vector field norm, interpolated on two cut planes immediately after the first bifucation (see Fig. 2), at peak inspiration $(t=0.4 s)$, transition $(t=1.6 s)$ and peak expiration $(t=1.9 \mathrm{~s})$.

We can observe that, in our experiments, the velocity profiles in these sections differ from inspiration and expiration and that during transition, as expected, more complex velocity profiles are formed in the upper airways. At the peak of inspiration we obtain a typical $M$-shape after the first bifurcation (see [13] where steady state simulations and experiments are performed on the same geometry).

Note that our numerical simulations are not able to capture the possible turbulent patterns of the flow since it will require a much more finer mesh and a parallel solver to perform DNS (Direct NavierStokes) simulations. Note moreover that in [9], [11], [14] the authors assume that the flow is laminar, at least for the respiration at rest. It seems to be due to the oscilatory character of the respiration which prevents the development of the turbulence patterns. Nevertheless, the geometries used to perform the simulations in the previous papers (as in the present work) go from the trachea up to few generations of the bronchial tree. It is worth noticing that a recent study [23], where steady state DNS simulations, with prescribed boundary conditions at the outlets and at the inlet, are performed, shows that for a geometry going from the mouth up to the to the 6th generation turbulence patterns appear. Whereas considering a geometry going from the trachea to the 6 th generation, for a steady flow, the turbulence is negligible. The model we propose could provide a tool to determine whether or not turbulent patterns appear in a pulsatile regime, by performing DNS simulation in a full geometry including the mouth-larynx system, with physically relevant driving forces, and free inlet boundary conditions. 


\section{Parametric studies}

In Figure 6 we compare the pressure field of the previous simulation at peak expiration with the case of a partially obstructed bronchial tree at the same time. The obstruction is obtained by increasing some resistance values (in this experiment, the resistances associated to the encircled outlets in Fig. 6 right). It shows that the qualitative behaviour of the flow is modified just by increasing few outlet resistances. Note that during an asthma crisis this is roughly what happens: the resistances of small airways in the distal part increase due to the inflammation process.
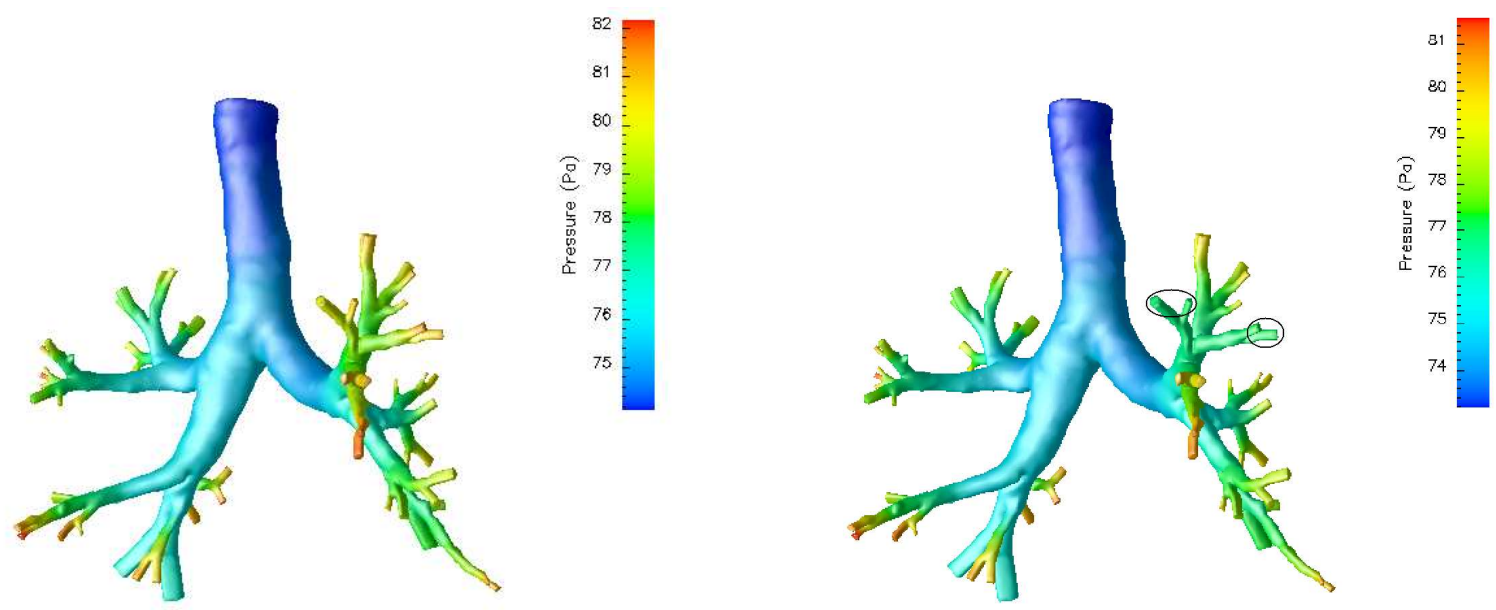

Figure 6: Pressure field in the standard (left) and obstructed (right) situations

Next we provide some experiments for forced respiration. In order to simulate the clinical experience of a normal subject performing forced maneuvers, we use a nonlinear constant $k=k(x)$ as in [25]. Indeed, in this case one needs to take into account the fact that the motion of the lung parenchyma is limited by the chest wall in order to capture the limitation of the inhaled volume. More precisely:

$$
k(x)=k_{0}+\left\{\begin{array}{l}
\left(f_{\min } / x_{\min }-k_{0}\right) x / x_{\min }, \text { if } x<0 \\
\left(f_{\max } / x_{\max }-k_{0}\right) x / x_{\max }, \text { if } x \geq 0 .
\end{array}\right.
$$

with $f_{\min }=-11 \times 10^{5} \mathrm{dyn}$ and $f_{\max }=13 \times 10^{5} \mathrm{dyn}$ and $k_{0}=3.63 \times 10^{3} \mathrm{dyn} \cdot \mathrm{cm}^{-1}$. The external force belongs to the following intervals:

$$
f_{\text {ext }} \in\left[-1.0 \times 10^{5}, 2.5 \times 10^{5}\right] \text { in } d y n \quad \text { (normal), } f_{\text {ext }} \in\left[-11 \times 10^{5}, 13 \times 10^{5}\right] \text { in dyn (forced). }
$$

The phase portrait (instantaneous flux vs. inhaled volume) is the curve obtained by spirometry, which provides pneumologists with information on the pathologies a patient may suffer. Figure 7 presents such a curve obtained by measurements, and Figure 8 the numerical results obtained after an appropriate tuning of the forcing term $f_{\text {ext }}$.

The model allows to investigate the influence of pertubations on the phase diagram, and thus to quantify the observability of different parameters with respect to this phase diagram. In this spirit, we can compute and compare phase diagrams obtained for different values of $k$ (see Fig. 9), and for different values of the distal resistance (Fig. 10). By these simulations we would like to emphasize 
that the coupled model we propose may be useful to reproduce some pathological features. A precise physiological exploration, in collaboration with lung specialists, still needs to be done.

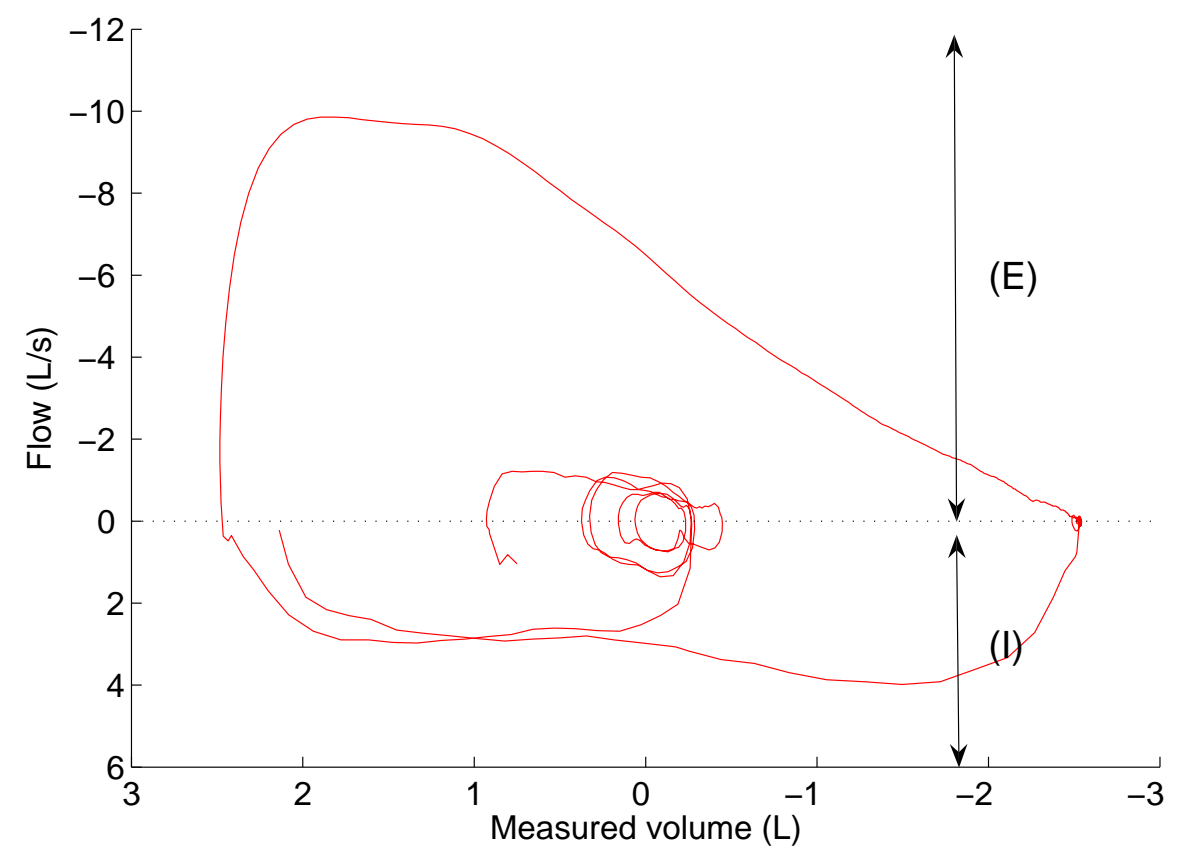

Figure 7: Measured Displacement (in $\mathrm{cm}$ )- Flow (in $\mathrm{cm}^{3} \cdot \mathrm{s}^{-1}$ ) diagram.

\section{Conclusions}

The proposed well-posed multiscale model enables to describe the air flow behavior in the proximal part of the bronchial tree taking into account the parenchyma and diaphram motions. We have studied the numerical feasibility of the model on a $3 D$ real geometry with realistic data. Note that in this whole coupled model we have only few parameters to fit: $m, k, S, f_{\text {ext }}$ and the resistances $R_{i}$. In particular by modifying $k$ and $R_{i}$ one can expect to reproduce some aspects of pathological behaviors such as asthma (increase of the resistances) or emphysema (decrease of $k$ ) by fitting to phase portrait and look, then, at the modifications induced for the $3 D$ flow. Nevertheless, we may have to modify the spring model to capture some of the non linear effects of the phenomenon and the physiological exploitation of this multiscale system will be the object of a forthcoming paper.

\section{A Hardy inequality in a quarter space}

We establish here a special Hardy inequality which is needed in the proof of Prop. 2.8. We consider the quarter space

$$
\Omega=\left\{x=\left(x_{1}, x_{2}, x_{3}\right)=\left(x^{\prime}, x_{3}\right) \in \mathbb{R}^{3}, x_{1} \geq 0, x_{2} \geq 0\right\} .
$$




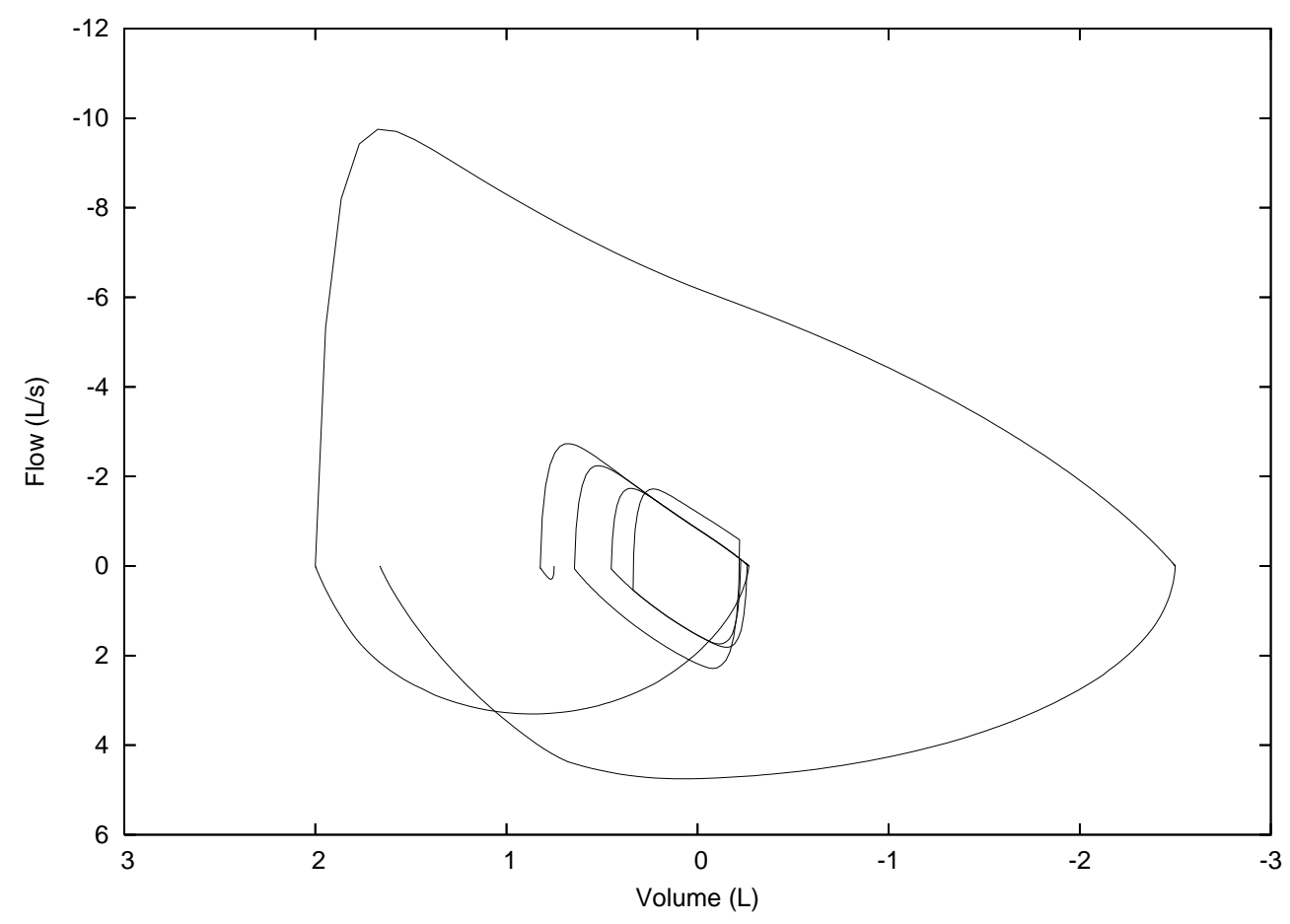

Figure 8: Displacement (in $\mathrm{cm}$ )- Flow (in $\mathrm{cm}^{3} \cdot \mathrm{s}^{-1}$ ) diagram.

For any $u$ in $L^{2}\left(\mathbb{R}^{3}\right)$, we denote by $\hat{u}$ its Fourier transform, we set $|u|_{s}^{2}=\int_{\mathbb{R}^{3}}|\xi|^{2 s}|\hat{u}(\xi)|^{2} d \xi$ and we denote by $\dot{H}^{s}\left(\mathbb{R}^{3}\right)$ the set of functions for which this quantity is bounded. The homogeneous Sobolev space $\dot{H}^{s}$ over $\Omega$ is defined as

$$
\dot{H}^{s}(\Omega)=\left\{u_{\mid \Omega}, u \in \dot{H}^{s}\left(\mathbb{R}^{3}\right)\right\},
$$

and it is endowed with the following semi-norm

$$
|u|_{\dot{H}^{s}(\Omega)}=\inf _{\tilde{u}_{\mid \Omega}=u}|\tilde{u}|_{s} .
$$

Finally, for $v$ defined over $\mathbb{R}^{d}$, sufficiently regular, we define $|D|^{\eta} v$ by

$$
\left(|D|^{\eta} v\right)(x)=(2 \pi)^{-d} \int_{\mathbb{R}^{d}} e^{i x \cdot \xi}|\xi|^{\eta} \hat{v}(\xi) d \xi
$$

Proposition A.1 Let $\delta \in(0,1 / 2)$ be given. There exists a constant $C>0$ such that, for any regular function $u$ which vanishes on $\left[x_{2}=0\right] \cap \partial \Omega$,

$$
|u|_{\dot{H}^{2-\delta}(\Omega)} \leq C\left\|\left|x^{\prime}\right|^{\delta} D^{2} u\right\|_{L^{2}(\Omega)},
$$

where $D^{2} u$ is the Hessian matrix of $u$, and $\left|x^{\prime}\right|=\left(x_{1}^{2}+x_{2}^{2}\right)^{1 / 2}$ is the distance to the $x_{3}$-axis. 


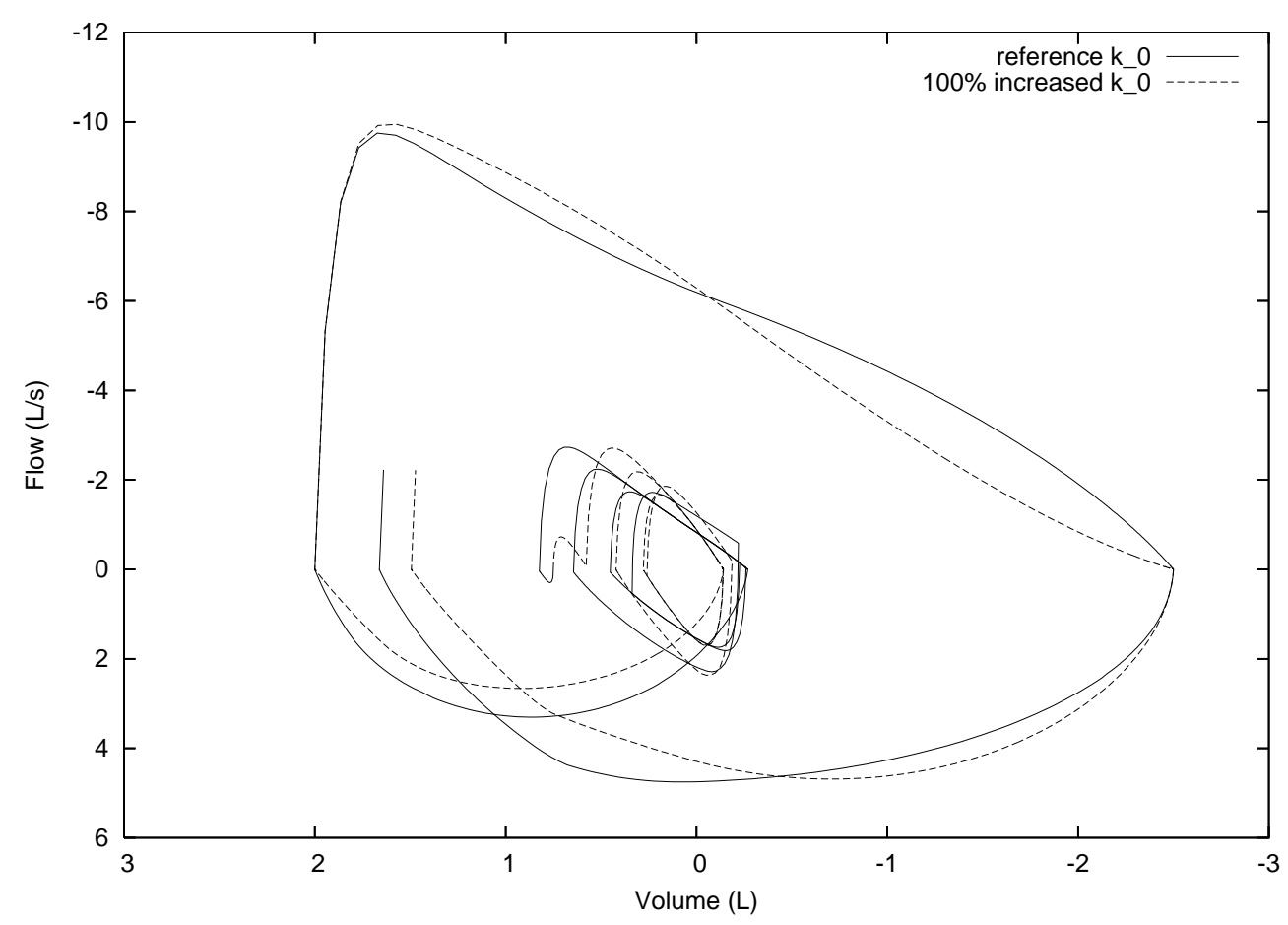

Figure 9: Phase diagram for different values of $k_{0}$

Proof: The proof decomposes into 4 steps.

a) Fractional Hardy inequality in $\mathbb{R}^{2}$

The standard Hardy inequality (which involves $u$ and its gradient) is also valid in the case fractional derivatives:

$$
\left\|\frac{u}{\left|x^{\prime}\right|^{\delta}}\right\|_{L^{2}\left(\mathbb{R}^{2}\right)} \leq=\left\||D|^{\delta} u\right\|_{L^{2}\left(\mathbb{R}^{2}\right)},
$$

for any function $u$ defined over $\mathbb{R}^{2}$, and $\delta \in(0,1)$. The proof is given in [37, 2].

b) Hardy inequality in $\mathbb{R}^{3}$, cylindrical setting

We prove here that the previous inequality extends to $\mathbb{R}^{3}$, with a weight equal to the distance $\left|x^{\prime}\right|$ to the $x_{3}$-axis. Let $u$ denote a regular function defined over $\mathbb{R}^{3}$. With obvious notations, one has

$$
\int_{\mathbb{R}^{3}} \frac{|u|^{2}}{\left|x^{\prime}\right|^{2 \delta}}=\int_{\mathbb{R}}\left(\int_{\mathbb{R}^{2}} \frac{|u|^{2}}{\left|x^{\prime}\right|^{2 \delta}} d x^{\prime}\right) d x_{3} \lesssim \int_{\mathbb{R}}\left\||D|^{\delta} u\right\|_{L^{2}\left(\left[x_{3}=s\right]\right)}^{2} d s=\left\||D|^{\delta} u\right\|_{L^{2}\left(\mathbb{R}^{3}\right)}^{2}
$$

c) Hardy inequality with a weight in the right-hand side

Let $u$ and $v$ be regular functions over $\mathbb{R}^{3}$. One has

$$
\left(|D|^{2-\delta} u, v\right)_{L^{2}\left(\mathbb{R}^{3}\right)}=\left(\left|x^{\prime}\right|^{\delta}|D|^{2} u, \frac{1}{\left|x^{\prime}\right|^{\delta}}|D|^{-\delta} v\right) \leq\left\|\left|x^{\prime}\right|^{\delta}|D|^{2} u\right\|_{L^{2}\left(\mathbb{R}^{3}\right)}\|v\|_{L^{2}\left(\mathbb{R}^{3}\right)}
$$




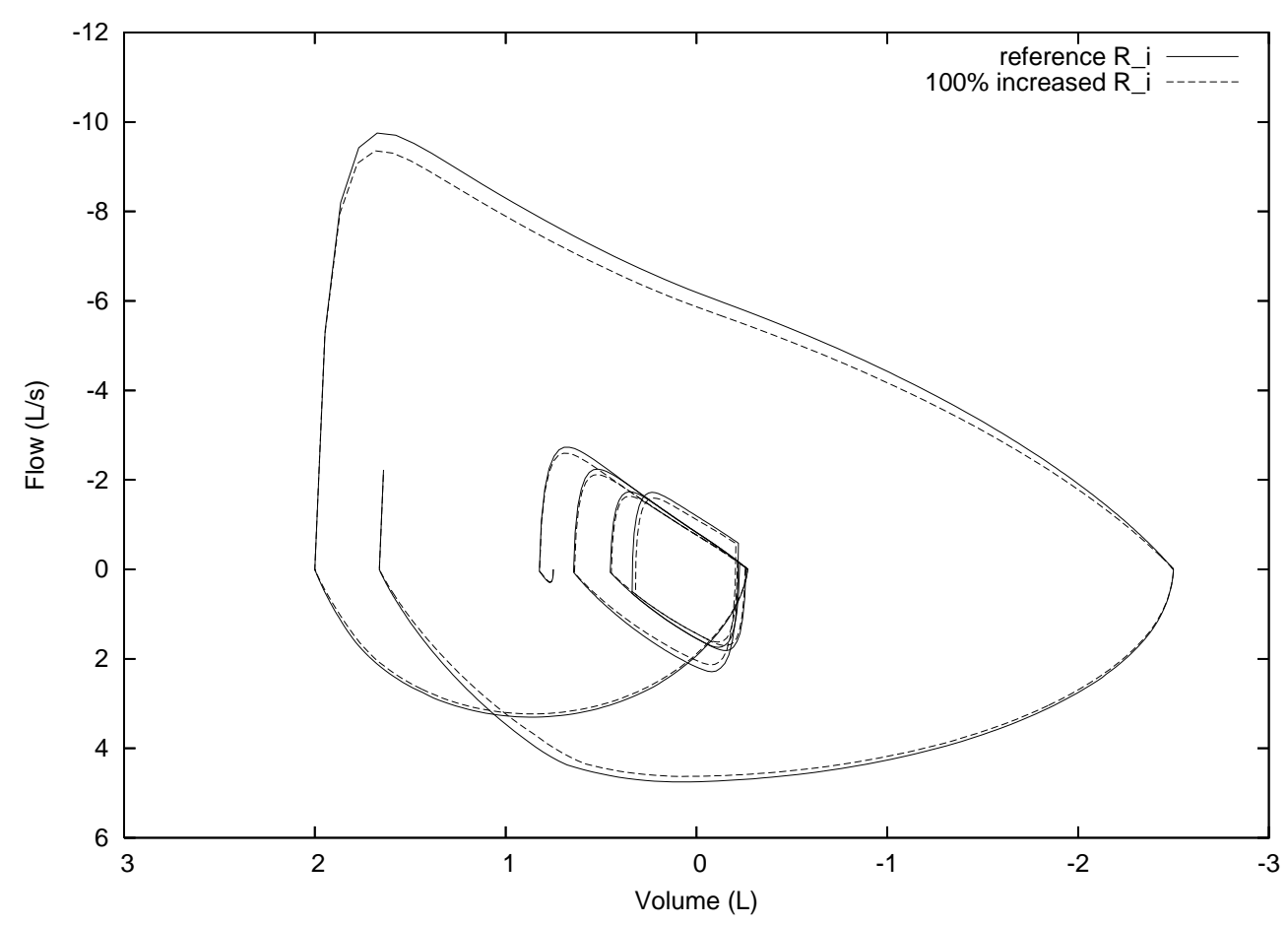

Figure 10: Phase diagram for different values of the distal resistances

(thanks to (43), which we apply to $|D|^{-\delta} v$ ). As a consequence, one has

$$
|u|_{\dot{H}^{2-\delta\left(\mathbb{R}^{3}\right)}} \lesssim\left\|\left|x^{\prime}\right|^{\delta} D^{2} u\right\|_{L^{2}\left(\mathbb{R}^{3}\right)} .
$$

\section{d) Hardy inequality in the quarter-space}

Let $u$ be a regular function defined over the quarter space $\Omega=\left[x_{1} \geq 0, x_{2} \geq 0\right]$. We assume that $u$ vanishes on $\left[x_{2}=0\right]$. We extend $u$ over $\mathbb{R}^{3}$ in two steps: firstly, we define $u_{1}$ over $\left[x_{1} \geq 0\right]$ as follows:

$$
u_{1}\left(x_{1}, x_{2}, x_{3}\right)=\mid \begin{array}{ll}
u\left(x_{1}, x_{2}, x_{3}\right) & \text { if } \quad x_{2} \geq 0 \\
-u\left(x_{1},-x_{2}, x_{3}\right) & \text { if } \quad x_{2} \leq 0
\end{array}
$$

this extension preserves the $L^{2}$ norm of $\left|x^{\prime}\right|^{\delta} D^{2} u$ (up to a factor 2 ). We extend now $u^{1}$ over $\mathbb{R}$ by following a Babitch process:

$$
u_{2}\left(x_{1}, x_{2}, x_{3}\right)=\mid \begin{array}{ll}
u_{1}\left(x_{1}, x_{2}, x_{3}\right) & \text { if } \quad x_{1} \geq 0 \\
3 u_{1}\left(-x_{1}, x_{2}, x_{3}\right)-2 u_{1}\left(-2 x_{1}, x_{2}, x_{3}\right) & \text { if } \quad x_{1} \leq 0
\end{array}
$$

This extension preserves again the $L^{2}$ norm of $\left|x^{\prime}\right|^{\delta} D^{2} u_{1}$ (up to a multiplicative constant). Inequality (44) can be applied to $u_{2}$, which ends the proof.

Acknowledgements The authors would like to acknowledge the support of ACINIM project LePoumonVousDisJe, and to thank M. Dauge for fruitful discussions, M. Grasseau for his technical help with the mesh, and P. Gérard for his help in proving Proposition A.1. 


\section{References}

[1] V. Antonaglia, L. Torelli, W.A. Zin and A. Gullo, Effects of viscoelasticity on volume distribution in a two-comportmental model of normal and sick lungs, Physiol. Meas. 26 (2005), 13-28.

[2] H. Bahouri, J.-Y. Chemin and I. Gallagher, Ingalités de Hardy précisées, C. R. Math. Acad. Sci. Paris 341 (2005), no. 2, 89-92.

[3] R. Begin, A.D. Renzetti Jr., A.H. Bigler and S. Watanabe, Flow and age dependence of airway closure and dynamic compliance, J. Appl. Physiol. 38 (1975), no 2, 199-207.

[4] A. Ben-Tal, Simplified models for gas exchange in the human lungs, J. Theor. Biol. 238 (2006), 474-495.

[5] W. Benish, P. Harper, J. Ward and J. Popovich, Jr., A mathematical model of lung static pressurevolume relationships: comparison of clinically derived parameters of elasticity, Henry Ford Hosp. Med. J. 36 (1988), no 1, 44-47.

[6] H. Brezis, Analyse Fonctionnelle, Théorie et applications, Ed. Dunod, 1994.

[7] G.P.S. Crooke, J.D. Head and J.J. Marini, A general two-compartment model for mechanical ventilation, Math. Comput. Modelling 24 (1996), no. 7, 1-18.

[8] Y.H. Chang and C.P. Yu, A model of ventilation distribution in the human lung, Aer. Sci. Tech. 30 (1999), 309-319.

[9] C. Croce, R. Fodil, M. Durand, G. Sbirlea-Apiou, G. Caillibotte, J.-F. Papon, J.-R. Blondeau, A. Coste, D. Isabey and B. Louis, In vitro experiments and numerical simulations of airflow in realistic nasal airway geometry Annals of biomedical engineering, (2006) Vol. 34, no6, pp. 997-1007.

[10] M. Dauge, Elliptic boundary value problems on corner domains, Smoothness and asymptotics of solutions, Lecture Notes in Mathematics, 1341. Springer-Verlag, Berlin, 1988.

[11] J.W. De Backer, W.G. Vos, A. Devolder, S.L. Verhulst, P. Germonpre, F.L. Wuyts, P.M. Parizel and W. De Backer Computational fluid dynamics can detect changes in airway resistance in asthmatics after acute bronchodilation, Journal of Biomechanics 41 (2008) 106-113.

[12] L. de Rochefort, X. Maître, R. Fodil, L. Vial, B. Louis, D. Isabey, C. Croce, L. Darrasse, G. Sbirlea-Apiou, G. Caillibotte, J. Bittoun and E. Durand, Phase contrast velocimetry with hyperpolarized helium-3 for in vitro and in vivo characterization of airflow Magn Reson Med 2006; $55: 1318-1325$.

[13] L. de Rochefort, L. Vial, R. Fodil, X. Matre, B. Louis, D. Isabey, G. Caillibotte, M. Thiriet, J. Bittoun, E. Durand and G. Sbirlea-Apiou, In vitro validation of computational fluid dynamic simulation in human proximal airways with hyperpolarized ${ }^{3}$ He magnetic resonance phase-contrast velocimetry, J. Appl Physiol, 102, pp. 2012-2023, 2007. 
[14] C. Fetita, S. Mancini, D. Perchet, F. Prêteux, M. Thiriet and L. Vial, An image based computational model of oscillatory flow in the proximal part of tracheobronchial trees, Comput. Meth. Biomech. Biomed. Eng., 8 (2005), pp. 27-293.

[15] L. Formaggia, F. Nobile, A. Quarteroni and A. Veneziani, Multiscale modelling of the circulatory system: A preliminary analysis, Comput. Visualization Sci. 2 (2/3) (1999) 7583.

[16] GHS3D, tetrahedal mesh generator, INRIA-Simulog, 2005 http://www-c.inria.fr/gamma/ghs3d

[17] V. Girault, P.-A. Raviart, Finite Element Approximation of the Navier-Stokes Equations, Lecture Notes in Mathematics, Vol. 749 Springer Verlag, Berlin, 1979.

[18] C. Grandmont, Y. Maday and B. Maury, A multiscale / multimodel approach of the respiration tree, Proceedings of the International Conference "New Trends in Continuum Mechanics" 8-12 September 2003, Constantza, Romania Theta Foundation Publications, Bucharest, 2005.

[19] C. Grandmont, B. Maury and N. Meunier, A viscoelastic model with non-local damping application to the human lungs, Math. Mod. Numer. Anal. 40 (2006), no 1, 201-224.

[20] C. Grandmont, B. Maury and A. Soualah, Multiscale modelling of the respiratory tract: a theoretical framework, ESAIM: PROCEEDINGS, June 2008, Vol. 23, p. 10-29

[21] J. G. Heywood, The Navier-Stokes equations : on the Existence, Regularity and decay of Solution, Indiana Univ. Math. J., 29, pp. 636-681 (1980).

[22] J. G. Heywood, R. Rannacher and S. Turek, Artificial boundaries and flux and pressure conditions for the incompressible Navier-Stokes equations, International Journal for Numerical Methods in Fluids, 22, pp. 325-352 (1996).

[23] C.-L. Lin, M. H. Tawhai, G. McLennanc and E.A. Hoffman Characteristics of the turbulent laryngeal jet and its effect on airflow in the human intra-thoracic airways, Respiratory Physiology \& Neurobiology, 157, pp. 295-309, (2007).

[24] J. L. Lions, Quelques méthodes de résolution des problèmes aux limites non linéaires, Dunod, 2002.

[25] S. Martin, B. Maury, T. Similowski and C. Straus, Modelling of respiratory system mechanics involving gas exchange in the human lungs, ESAIM: PROCEEDINGS, June 2008, Vol. 23, p. $30-47$.

[26] B. Maury, N. Meunier, A. Soualah and L. Vial, Outlet Dissipative conditions for air flow in the bronchial tree, ESAIM Proceedings, september 2005, vol. 14, 115-123, Eric Cancès \& JeanFrédéric Gerbeau, Editors.

[27] B. Mauroy, M. Filoche, J.S. Andrade Jr. and B. Sapoval, Interplay between flow distribution and geometry in an airway tree, Phys. Rev. Lett. 90, 14 (2003). 
[28] V. Maz'ya and J. Rossmann, Point estimates for Green's matrix to boundary value problems for second order elliptic systems in a polyhedral cone ZAMM Z. Angew. Math. Mech. 82 (2002), no. 5, 291-316.

[29] V. Maz'ya and J. Rossmann, $L_{p}$ estimates of solutions to mixed boundary value problems for the Stokes system in polyhedral domains, Math. Nachr. 280 (2007), no. 7, 751-793.

[30] M.S. Olufsen, Structured tree outflow condition for blood flow in larger systemic arteries, Am. J. Physiol. 276 (1999) 257-H268.

[31] Mathematical Models in Human Physiology, J.T. Ottesen, M.S. Olufsen, J.K. Larsen (editors), SIAM, Philadelphia, 2004.

[32] M. Orlt and A.-M. Sändig, Regularity of viscous Navier-Stokes flows in nonsmooth domains. Boundary value problems and integral equations in nonsmooth domains (Luminy, 1993), 185201, Lecture Notes in Pure and Appl. Math., 167, Dekker, New York, 1995.

[33] A. Quarteroni and A. Veneziani, Analysis of a Geometrical Multiscale Model Based on the Coupling of ODEs and PDEs for Blood Flow Simulations, Multiscale Model. Simul., Vol. 1, No. 2, 173-195, 2003.

[34] A. Quarteroni, S. Ragni and A. Veneziani, Coupling between lumped and distributed models for blood flow problems, Comput. Visualization Sci. 4 (2) (2001) 111-124.

[35] J.R. Rodarte and K. Rehder, Dynamic of respiration, In: Handbook of physiology: the respiratory system, A.P. Fishman, P.T. Macklem, J. Mead and S.R. Geiger (editors), Americal Physiological Society, 131-144, 1986.

[36] T. Similowski and J.H.T. Bates, Two-compartment modelling of respiratory system mechanics at low frequencies: gas redistribution or tissue rheology?, Eur. Respir. J. 4 (1991), 353-358.

[37] F. Vigneron Espaces de Sobolev d'ordre variable: traces, éclatement, inégalité de Hardy, Séminaire Équations aux dérivées partielles (Polytechnique) (2006-2007), Exp. No. 17.

[38] I. E. Vignon-Clementel, C. A. Figueroa, K. E. Jansen and C. A. Taylor, Outflow boundary conditions for three-dimensional finite element modelling of blood and pressure in arteries, Comput. Methods Appl. Mech. Engrg., 195, 3776-3796 , 2006.

[39] J.G. Venegas, R.S. Harris and B.A. Simon, A comprehensive equation for the pulmonary pressurevolume curve, J. Appl. Physiol. 81 (1998), no 1, 389-395.

[40] E.R. Weibel, Morphometry of the human lung, Springer Verlag and Academic Press, Berlin, New York, 151 pp., 1963.

[41] J.B. West, J.B. Respiratory Physiology - The Essentials, Baltimore: Williams \& Wilkins, 1974.

[42] The lung: scientific foundations, R.G. Crystal, J.B. West, E.R. Weibel and P.J. Barnes (editors), 2 Vol., 2nd Edition, Lippincott-Raven Press, Philadelphia, 1997. 